\title{
Article
}

\section{Parents' experiences of emotional closeness to their infants in the neonatal unit: A meta-ethnography}

Thomson, Gillian, Flacking, Renée, George, Kendall, Feeley, Nancy, Haslund-Thomsen, Helle, De Coen, Kris, Schmied, Virginia, Provenzi, Livio and Rowe, Jen

Available at http://clok.uclan.ac.uk/34693/

Thomson, Gillian ORCID: 0000-0003-3392-8182, Flacking, Renée, George, Kendall, Feeley, Nancy, Haslund-Thomsen, Helle, De Coen, Kris, Schmied, Virginia, Provenzi, Livio and Rowe, Jen (2020) Parents' experiences of emotional closeness to their infants in the neonatal unit: A meta-ethnography. Early Human Development, 149 . p. 105155. ISSN 0378-3782

It is advisable to refer to the publisher's version if you intend to cite from the work. http://dx.doi.org/10.1016/j.earlhumdev.2020.105155

For more information about UCLan's research in this area go to http://www.uclan.ac.uk/researchgroups/ and search for <name of research Group>.

For information about Research generally at UCLan please go to http://www.uclan.ac.uk/research/

All outputs in CLoK are protected by Intellectual Property Rights law, including Copyright law. Copyright, IPR and Moral Rights for the works on this site are retained by the individual authors and/or other copyright owners. Terms and conditions for use of this material are defined in the policies page. 


\begin{abstract}
Background: Physical and emotional parent-infant closeness activate important neurobiological mechanisms involved in parenting. In a neonatal care context, most research focuses on physical (parental presence, skin-to-skin contact) aspects; insights into emotional closeness can be masked by findings that overemphasise the barriers or challenges to parenting an infant during neonatal care.
\end{abstract}

Aim: To explore existing qualitative research to identify what facilitates and enables parents' experiences of emotional closeness to their infants while cared for in a neonatal unit.

Study design: A systematic review using meta-ethnographic methods. Search strategy involved searches on six databases, author runs, and backward and forward chaining. Reciprocal translation was used to identify and compare key concepts of parent-infant emotional closeness.

Results: Searches identified 6,992 hits, and 34 studies from 17 countries that involved 670 parents were included. Three overarching themes and associated sub-themes were developed. 'Embodied connections' describes how emotional closeness was facilitated by reciprocal parent-infant interactions, spending time as a family, and methods for parents to feel connected while physically separated. 'Inner knowing' concerns how knowledge about infant and maternal health and understanding the norms of neonatal care facilitated emotional closeness. 'Evolving parental role' relates to how emotional closeness was intertwined with parental identities of contributing to infant health, providing direct care, and being acknowledged as a parent.

Conclusion: Parent-infant closeness evolves and is facilitated by multifaceted biopsychosocial factors. Practice implications include creating private and uninterrupted family time, strategies for parents to maintain an emotional connection to their infant when separated, and neurobiology education for staff. 
Keywords: emotional closeness; neonatal care; meta-ethnography; parent-infant relationships; premature; NICU

\section{Introduction}

Globally, more than $10 \%$ of infants require admission to neonatal units (NUs) due to prematurity or ill health [1]. Much research has focused on how parents of infants admitted to a NU experience parenthood, and the emotional impact of having a sick and/or premature infant. A scoping review into the mental health of parents of infant in the NU highights that parents, regardless of culture and country, report feelings of guilt and shame, high levels of stress, and anxiety [2]. There are numerous triggers for these negative experiences including a stressful birth experience, and fears and concerns for the infant's wellbeing [3]. Parent-infant separation and the unfamiliar neonatal environment can lead to parents feeling estranged from their infant and lacking confidence in their parental role [3].

Over the last few decades, medical and nursing care in NUs has substantially improved. Family centred care was introduced to place families at the centre of the decision-making and caregiving processes and procedures for their infants, with positive and effective collaboration between professionals and family members being at the crux $[4,5]$. Previously, parents were only allowed to visit their infants on the NU for a few scheduled hours per day. Yet, while parents in many settings are now more present and involved in the care for their infant, the variations within and between countries are vast $[6,7]$. The impact of design of NUs on infants' and parents' wellbeing has also received increasing attention [8]. In particular, there is a trend towards replacing open bay design units with single family room design when building or renovating NUs [9]. The single family room design has been associated with more parental presence and skin-to-skin contact, and lower rates of stress and depression symptoms in parents $[10,11]$. 
Both physical and emotional closeness are considered essential for parent and infant health and wellbeing [12]. Physical closeness in a NU ranges from skin-to-skin contact between parentinfant, to parents being present in the unit but not in physical contact with their infant(s) [12]. Emotional closeness reflects psychological bonding [13], and ranges from strong and consistent to more distant feelings of love, care, affection and/or connection between the parent and the infant [12]. A large body of research has focused on the experiences and the barriers and facilitators for physical presence and contact within neonatal care (i.e., parental presence and skin-to-skin contact), for example $[6,7,11]$. However, there is limited knowledge on parental experiences of emotional closeness [14].

To date, a few studies have explored parent-infant closeness during the infant's admission on a NU [14-16]. One cross-national qualitative study involved parents completing an emotional closeness diary, and findings revealed that parents used multiple pathways - psychological, physical, spatial, relational, cognitive and social - that encouraged and enabled them to feel emotionally close to their infants [14]. Further insights into how emotional closeness can be facilitated in a NU are evident within a wider body of literature, such as studies focused on parents' experiences of forming relationships with their sick/premature infants [17-19], or of specific activities such as infant feeding [20, 21] or skin-to-skin/kangaroo care [22, 23]. However, these positive aspects can often be masked by overemphasising the barriers or challenges to parenting an infant during neonatal care. Aaron Antonovksy [24], a medical sociologist, coined the phrase 'salutogenesis' to argue that while a pathological focus was needed to understand the causes and impact of poor health, there needed to be greater emphasis and understanding on why and how individuals can remain in positive health, e.g., feeling emotional closeness with their infant during neonatal care. 
We adopted a salutogenic approach to explore the existing literature in order to develop new understandings of emotional closeness in a neonatal care context. As the experience and meanings of this phenomenon are likely best articulated in analytical concepts arising from themes or categories in qualitative or mixed-methods studies, a meta-ethnographic approach was adopted. The aim was to identify and synthesise existing qualitative-based literature to identify when, how and what situations and events enabled parents to feel emotionally close with their infants while cared for in a NU. A salutogenic perspective to uncover and illuminate how to facilitate parent-infant emotional closeness during neonatal care holds important implications for service delivery, and the potential to promote and optimize positive parent and infant health and wellbeing.

\section{Methodology}

Review methodology

A systematic review using meta-ethnographic methods to extract and analyse the findings was undertaken [25]. The meta-ethnographic approach was originally developed by Noblit and Hare [26] to synthesise the findings from ethnographic studies, but is now a widely used approach to combine and re-interpret findings from a range of qualitative methodological approaches [25]. The eMERGe Project was developed to improve the use and execution of meta-ethnographies [27] and their protocol for meta-ethnographic reporting [25] has been adhered to in the reporting of this review. The review protocol was uploaded and published in PROSPERO [28]. 


\section{Search strategy}

We used the PEO (Population; Exposure; Outcomes/themes) structure to identify search terms (see Table 1). The terms were developed following a priori scoping exercise (and adapted for specific database architecture) and were agreed in conjunction with a librarian at the lead author's university. An additional search string relating to 'study type' was also used in occasions where the number of hits exceeded $>1,000$. A schematic overview of the search string developed according to the PEO structure, the inclusion and exclusion criteria and additional selection criteria (date of publication, study type and language) is reported in Table 1.

Table 1: Search terms and inclusion/exclusion criteria mapped to PEO framework

\begin{tabular}{|c|c|c|c|}
\hline Criteria & Inclusion criteria & Exclusion criteria & Terms \\
\hline Study population & $\begin{array}{l}\text { Parents of infants who } \\
\text { had been admitted to } \\
\text { the neonatal unit } \\
\text { Parent = (mother, } \\
\text { father, parent, partner } \\
\text { [primary partner) } \\
\text { studies of both or either } \\
\text { Neonatal unit = NICU, } \\
\text { SCN neonatal nursery }\end{array}$ & $\begin{array}{l}\text { Parents of infants not } \\
\text { admitted to neonatal unit } \\
\text { Extended family }\end{array}$ & $\begin{array}{l}\text { Parent* } \\
\text { mother* or } \\
\text { partner* or } \\
\text { father* or famil* } \\
\text { or maternal or } \\
\text { paternal }\end{array}$ \\
\hline $\begin{array}{l}\text { Exposure in } \\
\text { context }\end{array}$ & $\begin{array}{l}\text { Parent experience, } \\
\text { perspective, in neonatal } \\
\text { unit setting }\end{array}$ & $\begin{array}{l}\text { Experiences, perspective } \\
\text { in setting not a neonatal } \\
\text { unit }\end{array}$ & $\begin{array}{lr}\text { "neonatal } & \\
\text { intensive" } & \text { or } \\
\text { neonatal } & \text { or } \\
\text { nursery } & \text { or } \\
\text { preterm } & \text { or } \\
\text { prematur* } & \text { or } \\
\text { "neonatal } & \text { unit" } \\
\text { or } \quad \text { neonatal } \\
\text { nursery" } \\
\text { "special or }\end{array}$ \\
\hline
\end{tabular}




\begin{tabular}{|c|c|c|c|}
\hline & & & $\begin{array}{l}\text { nursery" or } \\
\text { NICU }\end{array}$ \\
\hline Outcomes & $\begin{array}{l}\text { Data concerns } \\
\text { 'emotional closeness' - } \\
\text { parents' accounts of } \\
\text { intimacy, love and } \\
\text { affection for their } \\
\text { infants. }\end{array}$ & $\begin{array}{l}\text { Views/experiences not in } \\
\text { relation to parent-infant } \\
\text { emotional closeness }\end{array}$ & $\begin{array}{ll}\text { close* }^{*} & \text { or } \\
\text { connect* } & \text { or } \\
\text { relationship* } & \text { or } \\
\text { emotion* } & \text { or } \\
\text { lov* or tender* }^{*} & \text { or warmth or } \\
\text { attach* or bond* } \\
\text { or affect* or } \\
\text { intimac* }\end{array}$ \\
\hline Date & 1990 to present & Prior to 1990 & \\
\hline Study type & $\begin{array}{l}\text { Qualitative studies, } \\
\text { mixed-methods }\end{array}$ & $\begin{array}{l}\text { Purely quantitative based } \\
\text { studies, clinical case } \\
\text { studies, reviews, theses, } \\
\text { opinion pieces, grey } \\
\text { literature. }\end{array}$ & $\begin{array}{l}\text { Qualitative or } \\
\text { interview* or } \\
\text { "focus group*" } \\
\text { or ethnograph* } \\
\text { or } \\
\text { phenomenolog* } \\
\text { or narrative* or } \\
\text { "grounded } \\
\text { theory" }\end{array}$ \\
\hline Language & $\begin{array}{l}\text { English, Italian, } \\
\text { Swedish, Danish, } \\
\text { French }\end{array}$ & Any other languages & \\
\hline
\end{tabular}

Any study that reported parents' experiences of how different care practices and/or contexts of neonatal care could influence parent-infant emotional closeness were to be included. All types of neonatal care were included, e.g., 'neonatal nursery' for infants who do not require intensive care, and Level III NUs units for those with the highest level of need. No infants (e.g., sick and/or preterm or needing monitoring) or parents (e.g., age, health, situation) were excluded, and the intention was that potential variations were to be accounted for in the analysis. We used definitions from libraries (Oxford-English online dictionary, www.en.oxforddictionaries.com) and existing literature [14] to define emotional closeness as parents' accounts of intimacy, love and affection for their infants. All qualitative studies were 
to be included, i.e., exploratory descriptive, narrative, interview - thematic, case study, descriptive, phenomenology, grounded theory, ethnography as well as mixed-methods studies that include qualitative data. The linguistic background of the authors also allowed for the inclusion of original studies published in different languages (e.g., English, Danish, Swedish, Italian and French). In line with the justification provided in the meta-synthesis by Gibbs and colleagues [29], only studies published from 1990 onwards were to be included due to the technological changes in neonatal care, and the advent of family centred care[30].

We used a comprehensive multiple-search strategy that was conducted on six bibliographic databases: Cumulative Index of Nursing and Allied Health Literature (CINAHL), MEDLINE (Ovid), PsychArticles, Web of Science, Embase, and Global Index Medicus. These databases were chosen for their coverage of different perspectives and diverse discipline investigations on NU parents' experience (e.g., psychological, medical, nursing, educational approaches). Additional search methods involved author runs, citation tracking and reference checking.

\section{Study selection and appraisal}

Database searches were undertaken by four members of the review team. This involved each member searching different databases and screening titles/abstracts; all potentially relevant articles were highlighted and downloaded into one EndNote file and duplicates removed. All articles were then screened by at least two members of the review team against inclusion/exclusion criteria and papers identified for full text review. All full text reviews were divided up across all members of the review team, and each paper was read in full by two reviewers. Agreements for inclusion were made by consensus, and any disagreements regarding inclusion were discussed with a third reviewer. The initial database searches were undertaken in July and August 2018, and re-run in April 2020. 
All articles were quality appraised using the instrument originally developed by Walsh and Downe $[31,32]$. This framework was developed based on an in-depth review of existing qualitative assessment tools, and consideration of rigour [31] and is well utilised in systematic reviews in perinatal research, for example [33-35]. The framework assesses studies against pre-defined criteria, and then a score from A to D is allocated (Table 2). Only studies that score $\mathrm{C}$ or higher were included in the final analysis.

Table 2: Scoring criteria for quality appraisal [32]

\begin{tabular}{|c|c|}
\hline Grade & Description \\
\hline $\mathrm{A}$ & $\begin{array}{l}\text { No, or few flaws. The study credibility, transferability, dependability and } \\
\text { confirmability are high; }\end{array}$ \\
\hline B & $\begin{array}{l}\text { Some flaws, unlikely to affect the credibility, transferability, dependability } \\
\text { and/or confirmability of the study; }\end{array}$ \\
\hline $\mathrm{C}$ & $\begin{array}{l}\text { Some flaws that may affect the credibility, transferability, dependability and/or } \\
\text { confirmability of the study; }\end{array}$ \\
\hline $\mathrm{D}$ & $\begin{array}{l}\text { Significant flaws that are very likely to affect the credibility, transferability, } \\
\text { dependability and/or confirmability of the study. }\end{array}$ \\
\hline
\end{tabular}

Key data were extracted into a pre-designed proforma which included study aims/research question, methodology, sample size, participant characteristics, data collection methods, key findings/themes, and the quality appraisal rating.

\section{Strategy for data synthesis}

An inductive and interpretative meta-ethnography approach was used. This approach differentiates between first, second and third order data [25, 26]. First order relates to 
participant quotes, second order are the paper authors' interpretations, and third order concerns the new interpretations generated by the review team $[25,26]$. Meta-ethnography involves identifying issues and concepts at the second order level, with first order (quotes) used to substantiate the interpretations. The data is then subjected to some form of translation in generating the third order interpretations; reciprocal (identifying what was similar), refutational (identifying contradicting or disconfirming data) and a line of argument synthesis (an overarching summary of all key issues) $[25,26]$.

Consistent with the salutogenic approach, our review adopted a positive, strength-based perspective to understand emotional closeness. We were not concerned with contradictory data (e.g., times, events when parents do not feel emotionally close), nor were we intending to include all the experiences, challenges and negative aspects that parents can experience when their infants are in neonatal care. A reciprocal level of translation was therefore undertaken.

A common approach when undertaking a meta-ethnography is to identify an index paper that details the core issues related to the phenomenon under focus[36, 37], e.g., emotional closeness. The second order data is extracted from the index paper to develop an initial coding framework. The framework is then used to record the concepts and themes, from the remaining papers, with updates and revisions made as new insights emerge. We used the study by Flacking et al [14] to develop the coding framework and five members of the review team then extracted data from the remaining papers. Each paper was assigned to a lead reviewer (to extract the data) and a secondary reviewer (to check that all key issues had been recorded). The lead author then combined and uploaded all the codes and data extracts into MaxQDA qualitative data analysis software. Further interpretive work was then undertaken to synthesise the coded data into sub- 
themes and themes that represented the whole data set. The final themes and sub-themes were reviewed, refined and agreed by all authors.

\section{Findings}

In the initial search 5,147 hits were retrieved from the database searches, and a further 1,775 during the updated searches. Eight papers were also identified via additional search methods. Four hundred and 28 articles were screened against inclusion/exclusion criteria, 115 were reviewed as full-texts and 34 included in the final review (see PRISMA, Figure 1).

The study characteristics and quality appraisal grade of the included studies are reported in Table 3. Overall these studies represent the views of 670 parents (467 mothers, 180 fathers and one study stating 23 parents); 17 studies focused on mothers, seven on fathers, and ten included both. Parental age ranged from 16- 46 years for mothers and 18-59 years for fathers, with most studies including a mix of parities. Twenty-seven studies were undertaken in highincome countries (USA $(n=8)$, Sweden $(n=4)$, Canada $(n=3)$, Australia $(n=2)$, Norway $(n=1)$, Taiwan $(n=1)$, UK $(n=1)$, France $(n=1)$, Denmark $(n=1)$, Finland $(n=1)$, Spain $(n=1)$, Italy $(n=1)$; six in upper-middle countries (South Africa $(n=2)$, Thailand $(n=1)$, Colombia $(n=2)$, Iran $(n=1)$, and one in a low income setting (Malawi). Three studies were undertaken in a neonatal nursery, and 25 in units that provided more acute and intensive care (6 unspecified). The age of the infant (where known) at the time of data collection ranged from 1 day to 5 years, and gestational age at birth varied from 24 to $>37$ weeks. Most studies were undertaken after $2010 \quad(n=23)$ and employed different methodological designs, e.g., qualitative descriptive, phenomenology, grounded theory. While most involved some form of interview, additional methods such as audio-recorded parent diaries, observations of parents in the NU, 
and field notes were also used. Thirteen studies were focused on parents' views of infant feeding ( $n=3)$, skin-to-skin/kangaroo care $(n=7)$ or a specific intervention (e.g., NIDCAP or singing, $n=3)$, and the remainder $(n=21)$ concerned with more general parental accounts of having a premature/sick infant cared for in a neonatal unit.

\section{Figure 1: PRISMA Flow Diagram}
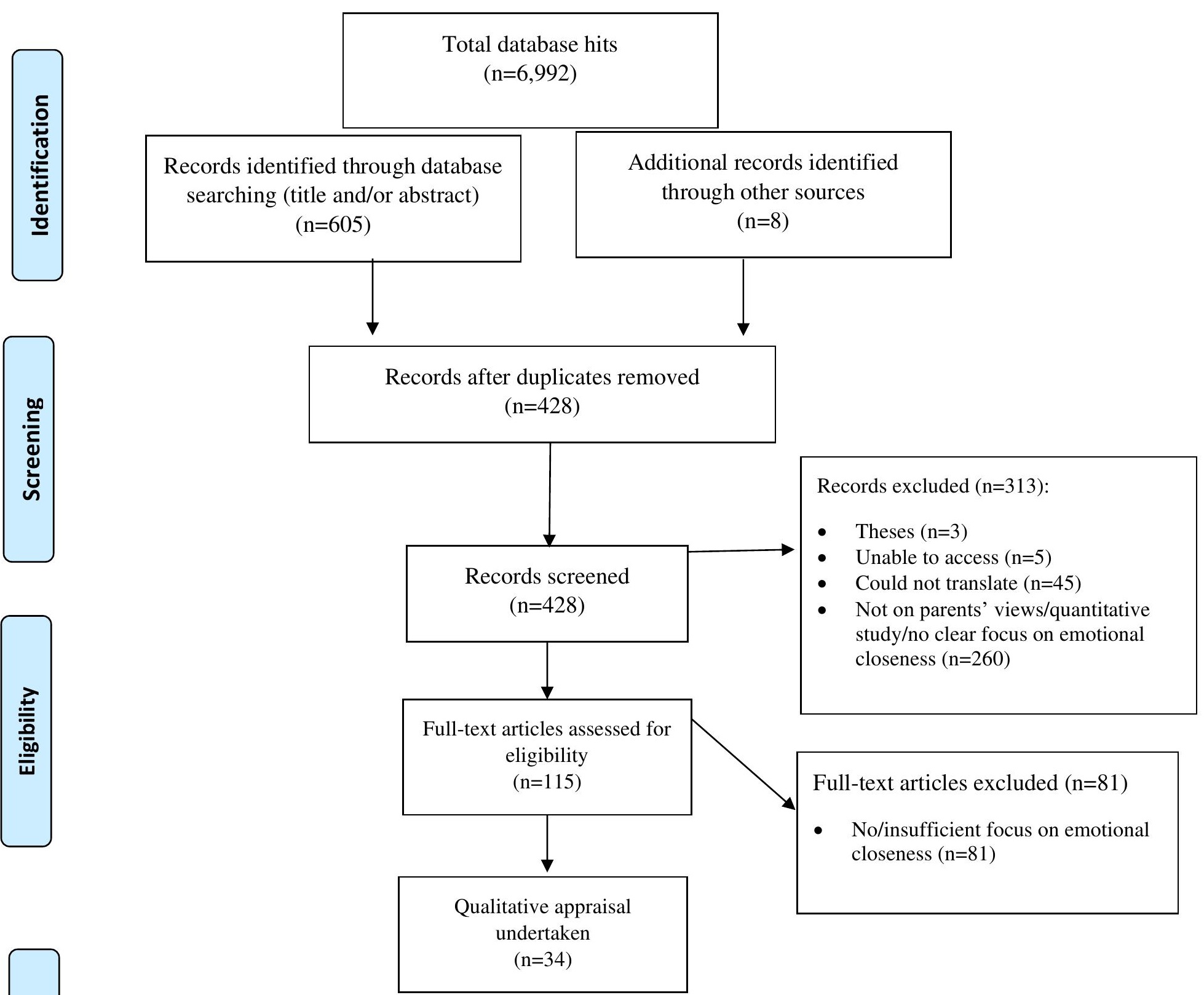


\begin{tabular}{|c|}
\hline \\
Studies included in \\
qualitative synthesis \\
$(\mathrm{n}=34)$
\end{tabular}


Table 3: Study characteristics and quality appraisal rating for all included studies $(n=34)$

\begin{tabular}{|c|c|c|c|c|c|c|c|c|c|c|}
\hline $\begin{array}{l}\text { Author } \\
\text { Year }\end{array}$ & Aim & $\begin{array}{l}\text { Country } \\
\text { Type of unit } \\
\text { single/multi-centre }\end{array}$ & Study design & $\begin{array}{l}\text { Age of } \\
\text { infant when } \\
\text { parent } \\
\text { participating } \\
\text { in the study }\end{array}$ & $\begin{array}{l}\text { Infants' GA } \\
\text { at birth }\end{array}$ & Sample & $\begin{array}{l}\text { Parent } \\
\text { characteristics } \\
\text { (age/parity) }\end{array}$ & $\begin{array}{l}\text { Data collection } \\
\text { methods }\end{array}$ & $\begin{array}{l}\text { Data analysis } \\
\text { methods }\end{array}$ & $\begin{array}{l}\text { QA } \\
\text { GRADE }^{1}\end{array}$ \\
\hline $\begin{array}{l}\text { Kavanaugh } \\
\text { et al } \\
1997[38]\end{array}$ & $\begin{array}{l}\text { Examine } \\
\text { rewards and } \\
\text { efforts of } \\
\text { breastfeeding } \\
\text { for mothers of } \\
\text { preterm infants. }\end{array}$ & $\begin{array}{l}\text { USA } \\
\text { NICU level III } \\
\text { single centre }\end{array}$ & Naturalistic inquiry & $\begin{array}{l}\text { After } \\
\text { discharge } \\
(1 \mathrm{~m})\end{array}$ & $\begin{array}{l}28 w-37 w \\
\text { Mean 32w4d }\end{array}$ & 20 mothers & $\begin{array}{l}\text { Mean age } 31.5 \\
\text { yrs; parity } \\
\text { unspecified }\end{array}$ & $\begin{array}{l}\text { Semi- } \\
\text { structured } \\
\text { interviews }\end{array}$ & $\begin{array}{l}\text { Within and } \\
\text { cross case } \\
\text { analysis of } \\
\text { codes }\end{array}$ & $\mathrm{B}+/ \mathrm{C}$ \\
\hline $\begin{array}{l}\text { Neu } \\
\text { 1999[39] }\end{array}$ & $\begin{array}{l}\text { Explore } \\
\text { parents' } \\
\text { perception of } \\
\text { and decisive } \\
\text { factors of } \\
\text { engaging into } \\
\text { skin-to-skin } \\
\text { care with } \\
\text { preterm infant } \\
\text { while on }\end{array}$ & $\begin{array}{l}\text { USA } \\
\text { NICU level II/III } \\
\text { single centre }\end{array}$ & Naturalistic inquiry & $\begin{array}{l}\text { Combination } \\
\text { in hospital } \\
\text { and after } \\
\text { discharge } \\
(4-17 \mathrm{~m})\end{array}$ & $\begin{array}{l}\text { Mean } 27.2 \mathrm{w} \\
\pm 2 \mathrm{w}\end{array}$ & $\begin{array}{l}8 \text { mothers } \\
1 \text { father }\end{array}$ & $\begin{array}{l}21-37 y ; 4 \text { first } \\
\text { time parents }\end{array}$ & $\begin{array}{l}\text { Interview, } \\
\text { Video } \\
\text { observation, } \\
\text { Field notes }\end{array}$ & $\begin{array}{l}\text { Thematic } \\
\text { analysis }\end{array}$ & $\mathrm{C}-$ \\
\hline
\end{tabular}




\begin{tabular}{|c|c|c|c|c|c|c|c|c|c|c|}
\hline & $\begin{array}{l}\text { assisted } \\
\text { ventilation }\end{array}$ & & & & & & & & & \\
\hline $\begin{array}{l}\text { Lupton \& } \\
\text { Fenwick } \\
\text { 2001[40] }\end{array}$ & $\begin{array}{l}\text { How do NICU } \\
\text { mothers } \\
\text { practice } \\
\text { motherhood and } \\
\text { construct } \\
\text { themselves as } \\
\text { mothers? }\end{array}$ & $\begin{array}{l}\text { Australia } \\
\text { NICU unspecified } \\
\text { multi-centre }\end{array}$ & $\begin{array}{l}\text { Unspecified } \\
\text { qualitative }\end{array}$ & $\begin{array}{l}\text { In hospital } \\
(3-14 w)\end{array}$ & $24 w->37 w$ & $\begin{array}{l}31 \text { mothers } \\
20 \text { nurses }\end{array}$ & $\begin{array}{l}\text { 19-41 years } \\
\text { (mean 28.2); } \\
21 \text { primips }\end{array}$ & $\begin{array}{l}\text { In-depth } \\
\text { interviews, } \\
\text { recorded dyad } \\
\text { (mother/nurse) } \\
\text { observations, } \\
\text { field notes }\end{array}$ & $\begin{array}{l}\text { Unspecified - } \\
\text { recurring } \\
\text { patterns in the } \\
\text { discourse }\end{array}$ & $\mathrm{A} / \mathrm{B}+$ \\
\hline $\begin{array}{l}\text { Roller } \\
\text { 2005[41] }\end{array}$ & $\begin{array}{l}\text { Understand } \\
\text { mothers' } \\
\text { experiences of } \\
\text { providing KMC } \\
\text { for their } \\
\text { preterm infant. }\end{array}$ & $\begin{array}{l}\text { USA } \\
\text { NICU unspecified } \\
\text { single centre }\end{array}$ & $\begin{array}{l}\text { Transcendental } \\
\text { phenomenology }\end{array}$ & $\begin{array}{l}\text { After } \\
\text { discharge } \\
(1-4 w)\end{array}$ & $32 w-37 w$ & 10 mothers & $\begin{array}{l}\text { Age unspecified } \\
\text { (4 teenagers); } \\
9 \text { primips }\end{array}$ & $\begin{array}{l}\text { Semi- } \\
\text { structured } \\
\text { interviews }\end{array}$ & $\begin{array}{l}\text { Thematic } \\
\text { content } \\
\text { analysis }\end{array}$ & $\mathrm{B}+$ \\
\hline $\begin{array}{l}\text { Johnson } \\
\text { 2007[23] }\end{array}$ & $\begin{array}{l}\text { Describe } \\
\text { maternal } \\
\text { experience with } \\
\text { KMC }\end{array}$ & \begin{tabular}{|l|} 
USA \\
NICU level III \\
single centre
\end{tabular} & $\begin{array}{l}\text { Qualitative } \\
\text { naturalistic inquiry }\end{array}$ & $\begin{array}{l}\text { In hospital } \\
\text { ( } 2 \mathrm{w} \text { after } \\
\text { birth) }\end{array}$ & $\begin{array}{l}\text { Mean GA } \\
28 \mathrm{w} 6 \mathrm{~d}\end{array}$ & 18 mothers & $\begin{array}{l}\text { Mean age } 26.3 y \text {; } \\
\text { All primips }\end{array}$ & $\begin{array}{l}\text { Interviews, } \\
\text { observations, } \\
\text { field notes }\end{array}$ & $\begin{array}{l}\text { Content } \\
\text { analysis }\end{array}$ & $\mathrm{B} / \mathrm{B}-$ \\
\hline
\end{tabular}




\begin{tabular}{|c|c|c|c|c|c|c|c|c|c|c|}
\hline & $\begin{array}{l}\text { of premature } \\
\text { infants in NICU }\end{array}$ & & & & & & & & & \\
\hline $\begin{array}{l}\text { Lundqvist et } \\
\text { al } \\
2007[42]\end{array}$ & $\begin{array}{l}\text { Describe } \\
\text { fathers' lived } \\
\text { experience of } \\
\text { caring for their } \\
\text { preterm infant. }\end{array}$ & $\begin{array}{l}\text { Sweden } \\
\text { NICU level II } \\
\text { single centre }\end{array}$ & $\begin{array}{l}\text { Hermeneutic } \\
\text { phenomenoloy }\end{array}$ & $\begin{array}{l}\text { Combination } \\
\text { in hospital } \\
\text { and after } \\
\text { discharge } \\
\text { (1-3m after } \\
\text { birth) }\end{array}$ & $25 w-32 w$ & 13 fathers & $\begin{array}{l}27-45 y ; 8 \text { first } \\
\text { time fathers }\end{array}$ & $\begin{array}{l}\text { Semi- } \\
\text { structured } \\
\text { interviews }\end{array}$ & $\begin{array}{l}\text { Thematic } \\
\text { analysis }\end{array}$ & $\mathrm{A} / \mathrm{B}$ \\
\hline $\begin{array}{l}\text { Orapiriyakul } \\
\text { et al } \\
2007[43]\end{array}$ & $\begin{array}{l}\text { Describe the } \\
\text { development of } \\
\text { maternal } \\
\text { attachment to } \\
\text { their preterm } \\
\text { infant while in } \\
\text { NICU. }\end{array}$ & $\begin{array}{l}\text { Thailand } \\
\text { NICU level III } \\
\text { multicentre }\end{array}$ & Grounded theory & $\begin{array}{l}\text { In hospital } \\
(2-33 d)\end{array}$ & $26 w-33 w$ & 15 mothers & $\begin{array}{l}\text { age } 16-41 \text { y; } 5 \\
\text { primips }\end{array}$ & $\begin{array}{l}\text { Semi- } \\
\text { structured } \\
\text { interviews, } \\
\text { video } \\
\text { observations }\end{array}$ & $\begin{array}{l}\text { Constant } \\
\text { comparative } \\
\text { analysis }\end{array}$ & $\mathrm{B} / \mathrm{C}$ \\
\hline $\begin{array}{l}\text { Fegran et al } \\
2008[44]\end{array}$ & $\begin{array}{l}\text { Obtain } \\
\text { knowledge of } \\
\text { and compare } \\
\text { parents' } \\
\text { individual } \\
\text { experiences of } \\
\text { the attachment } \\
\text { process }\end{array}$ & $\begin{array}{l}\text { Norway } \\
\text { NICU unspecified } \\
\text { single centre }\end{array}$ & $\begin{array}{l}\text { Descriptive with a } \\
\text { hermenutic } \\
\text { approach }\end{array}$ & $\begin{array}{l}\text { around } \\
\text { discharge } \\
(3 \mathrm{~d}-14 \mathrm{w})\end{array}$ & $28 w-32 w$ & $\begin{array}{l}6 \text { mothers } \\
6 \text { fathers }\end{array}$ & $\begin{array}{l}\text { Mothers age 26- } \\
\text { 42y; } 2 \text { primips } \\
\text { Fathers age } 27- \\
59 y \text {. }\end{array}$ & $\begin{array}{l}\text { Semi- } \\
\text { structured } \\
\text { interviews }\end{array}$ & $\begin{array}{l}\text { Kvale's (1996) } \\
\text { contexts of } \\
\text { interpreting } \\
\text { qualitative } \\
\text { data. }\end{array}$ & $\mathrm{B} / \mathrm{B}+$ \\
\hline
\end{tabular}




\begin{tabular}{|c|c|c|c|c|c|c|c|c|c|c|}
\hline & $\begin{array}{l}\text { immediately } \\
\text { after a } \\
\text { premature birth. }\end{array}$ & & & & & & & & & \\
\hline $\begin{array}{l}\text { Fenwick et } \\
\text { al } \\
2008[19]\end{array}$ & $\begin{array}{l}\text { Understand } \\
\text { how women } \\
\text { begin their roles } \\
\text { as mothers } \\
\text { when their } \\
\text { infant is in the } \\
\text { neonatal } \\
\text { nursery. }\end{array}$ & $\begin{array}{l}\text { Australia } \\
\text { neonatal nursery } \\
\text { multi-centre }\end{array}$ & Grounded theory & $\begin{array}{l}\text { Combination } \\
\text { in hospital } \\
\text { and after } \\
\text { discharge } \\
(8-12 w)\end{array}$ & Unspecified & $\begin{array}{l}28 \text { mothers } \\
20 \text { staff }\end{array}$ & $\begin{array}{l}\text { Mean age } 28.2 y \text {; } \\
\text { parity } \\
\text { unspecified. }\end{array}$ & $\begin{array}{l}\text { Semi- } \\
\text { structured } \\
\text { interviews, } \\
\text { observations } \\
\text { field notes. }\end{array}$ & $\begin{array}{l}\text { Constant } \\
\text { comparative } \\
\text { method }\end{array}$ & $\mathrm{A} / \mathrm{B}$ \\
\hline $\begin{array}{l}\text { Leonard \& } \\
\text { Mayers } \\
\text { 2008[22] }\end{array}$ & $\begin{array}{l}\text { Describe the } \\
\text { lived } \\
\text { experience of } \\
\text { KMC with } \\
\text { preterm infant }\end{array}$ & $\begin{array}{l}\text { South Africa } \\
\text { neonatal nursery } \\
\text { single centre }\end{array}$ & Phenomenology & $\begin{array}{l}\text { Combination } \\
\text {, } 5 \text { in } \\
\text { hospital/ } 1 \\
\text { after } \\
\text { discharge } \\
\text { (all 1w after } \\
\text { birth) }\end{array}$ & $<37 \mathrm{w}$ & $\begin{array}{l}4 \text { mothers } \\
2 \text { fathers }\end{array}$ & Unspecified & $\begin{array}{l}\text { In-depth } \\
\text { interviews }\end{array}$ & $\begin{array}{l}\text { Adapted } \\
\text { thematic } \\
\text { approach }\end{array}$ & $\mathrm{C}$ \\
\hline
\end{tabular}




\begin{tabular}{|c|c|c|c|c|c|c|c|c|c|c|}
\hline $\begin{array}{l}\text { Chang Lee } \\
\text { et al } \\
2009[45]\end{array}$ & $\begin{array}{l}\text { Examine } \\
\text { parenting } \\
\text { experiences of } \\
\text { mothers during } \\
\text { hospitalisation } \\
\text { of VLBW } \\
\text { preterm infants } \\
\text { under Chinese } \\
\text { custom of one } \\
\text { month maternal } \\
\text { rest. }\end{array}$ & $\begin{array}{l}\text { Taiwan } \\
\text { NICU level II/III } \\
\text { single centre }\end{array}$ & Grounded theory & $\begin{array}{l}\text { In hospital } \\
\text { (unspecified) }\end{array}$ & $\begin{array}{l}25 \mathrm{w}-34 \mathrm{w} \\
\text { mean }=28 \mathrm{w} 2 \\
\text { d }\end{array}$ & 26 mothers & $\begin{array}{l}22-36 \text { y; } 9 \\
\text { primips }\end{array}$ & $\begin{array}{l}\text { In-depth } \\
\text { interviews, } \\
\text { observations }\end{array}$ & $\begin{array}{l}\text { Constant } \\
\text { comparative } \\
\text { method }\end{array}$ & $\mathrm{B}+/ \mathrm{B}$ \\
\hline $\begin{array}{l}\text { Blomqvist et } \\
\text { al } \\
2012[46]\end{array}$ & $\begin{array}{l}\text { Describe } \\
\text { father's } \\
\text { experiences } \\
\text { with KMC of } \\
\text { their preterm } \\
\text { infants. }\end{array}$ & $\begin{array}{l}\text { Sweden } \\
\text { NICU level III } \\
\text { multi-centre }\end{array}$ & $\begin{array}{l}\text { Qualitative } \\
\text { descriptive }\end{array}$ & $\begin{array}{l}\text { After } \\
\text { discharge } \\
(4 \mathrm{~m} \\
\text { corrected } \\
\text { age })\end{array}$ & $\begin{array}{l}29 w 3 d-33 w \\
3 d\end{array}$ & 7 fathers & $\begin{array}{l}25-36 y ; \\
\text { all first time } \\
\text { father }\end{array}$ & $\begin{array}{l}\text { Semi- } \\
\text { structured } \\
\text { interviews }\end{array}$ & $\begin{array}{l}\text { Qualitative } \\
\text { content } \\
\text { analysis }\end{array}$ & $\mathrm{A}+\mathrm{B}$ \\
\hline $\begin{array}{l}\text { Arnold et al } \\
\text { 2013[47] }\end{array}$ & $\begin{array}{l}\text { Explore parents' } \\
\text { initial } \\
\text { experiences of } \\
\text { the birth of their } \\
\text { very preterm } \\
\text { baby and their }\end{array}$ & $\begin{array}{l}\text { England } \\
\text { NICU level III } \\
\text { multi-centre }\end{array}$ & Qualitative study & $\begin{array}{l}\text { Combination } \\
\text { in hospital or } \\
\text { after } \\
\text { discharge } \\
\text { (44-344 days } \\
\text { - mean } 154 \\
\text { days) }\end{array}$ & $\begin{array}{l}24 \mathrm{w}-32 \mathrm{w} \\
(\text { mean } \\
29 \mathrm{w} 2 \mathrm{~d} \pm \\
2 \mathrm{w})\end{array}$ & $\begin{array}{l}32 \text { mothers } \\
7 \text { fathers }\end{array}$ & $\begin{array}{l}\text { Age } 25-44 \text { yrs; } \\
75 \% \text { primips }\end{array}$ & $\begin{array}{l}\text { Semi- } \\
\text { structured } \\
\text { interviews }\end{array}$ & $\begin{array}{l}\text { Inductive } \\
\text { systematic } \\
\text { thematic } \\
\text { analysis }\end{array}$ & $\mathrm{B}+/ \mathrm{C}+$ \\
\hline
\end{tabular}




\begin{tabular}{|c|c|c|c|c|c|c|c|c|c|c|}
\hline & $\begin{array}{l}\text { stay in the } \\
\text { NICU. }\end{array}$ & & & & & & & & & \\
\hline $\begin{array}{l}\text { Guillaume et } \\
\text { al } \\
2013[18]\end{array}$ & $\begin{array}{l}\text { Explore parents' } \\
\text { early bonding } \\
\text { with their very } \\
\text { preterm infant, } \\
\text { and parent's } \\
\text { expectations of }\end{array}$ & \begin{tabular}{|l} 
France \\
NICU level III \\
multi-centre
\end{tabular} & Discourse analysis & $\begin{array}{l}\text { In hospital } \\
\text { ( } 24 \mathrm{~d} \pm 10 \mathrm{~d} \\
\text { after birth) }\end{array}$ & $\begin{array}{l}\text { Mean } 27 \mathrm{w} \\
\pm 2 \mathrm{w}\end{array}$ & $\begin{array}{l}30 \text { mothers } \\
30 \text { fathers }\end{array}$ & $\begin{array}{l}\text { Mothers age } \\
30,7 y \pm 6,6 ; \\
\text { Fathers age } 33,5 y \\
\pm 6,8 ; \\
\text { parity } \\
\text { unspecified }\end{array}$ & $\begin{array}{l}\text { Semi- } \\
\text { structured } \\
\text { interviews }\end{array}$ & $\begin{array}{l}\text { Horizontal and } \\
\text { vertical } \\
\text { (discourse) } \\
\text { analysis }\end{array}$ & $\mathrm{A} / \mathrm{B}$ \\
\hline $\begin{array}{l}\text { Rossman et } \\
\text { al } \\
2013[48]\end{array}$ & $\begin{array}{l}\text { Describe the } \\
\text { meaning of } \\
\text { milk for } \\
\text { mothers of } \\
\text { VLBW } \\
(<1500 g) \\
\text { infants in the } \\
\text { NICU }\end{array}$ & \begin{tabular}{|l|} 
USA \\
NICU level III \\
single centre
\end{tabular} & $\begin{array}{l}\text { Qualitative } \\
\text { descriptive }\end{array}$ & $\begin{array}{l}\text { In hospital } \\
\text { (4-8w after } \\
\text { birth) }\end{array}$ & $\begin{array}{l}23 w-33 w \\
\text { mean } 26 w 6 d\end{array}$ & 23 mothers & $\begin{array}{l}\text { age 19-37y } \\
\text { (mean 26y); } \\
78 \% \text { primips }\end{array}$ & $\begin{array}{l}\text { In-depth } \\
\text { interviews, } \\
\text { observations, } \\
\text { field notes }\end{array}$ & $\begin{array}{l}\text { Inductive } \\
\text { content } \\
\text { analysis }\end{array}$ & $\mathrm{B}+/ \mathrm{C}$ \\
\hline $\begin{array}{l}\text { Flacking \& } \\
\text { Dykes } \\
\text { 2013[49] }\end{array}$ & $\begin{array}{l}\text { Explore the } \\
\text { impact of place } \\
\text { and space on } \\
\text { parents' }\end{array}$ & $\begin{array}{l}\text { Sweden/England } \\
\text { NICU level II/III } \\
\text { multi-centre }\end{array}$ & Ethnography & $\begin{array}{l}\text { In hospital } \\
\text { (unspecified) }\end{array}$ & $25 w-35 w$ & $\begin{array}{l}52 \text { mothers } \\
19 \text { fathers } \\
102 \text { staff }\end{array}$ & $\begin{array}{l}\text { Mothers age } 22- \\
45 y \text {; } 30 \text { primips } \\
\text { Fathers age } 23- \\
47 y\end{array}$ & $\begin{array}{l}\text { Interviews, } \\
\text { observations, } \\
\text { field notes }\end{array}$ & $\begin{array}{l}\text { Grounded } \\
\text { theory }\end{array}$ & $\mathrm{A} / \mathrm{B}$ \\
\hline
\end{tabular}




\begin{tabular}{|c|c|c|c|c|c|c|c|c|c|c|}
\hline & $\begin{array}{l}\text { experiences and } \\
\text { practices related } \\
\text { to feeding their } \\
\text { preterm babies } \\
\text { in NICUs. }\end{array}$ & & & & & & & & & \\
\hline $\begin{array}{l}\text { Ettenberger } \\
\text { et al } \\
2014[50]\end{array}$ & $\begin{array}{l}\text { To extend the } \\
\text { existing } \\
\text { knowledge } \\
\text { about music } \\
\text { therapy in the } \\
\text { NICU and to } \\
\text { provide new } \\
\text { insights by } \\
\text { using live } \\
\text { music }\end{array}$ & $\begin{array}{l}\text { Colombia } \\
\text { NICU level III } \\
\text { single centre }\end{array}$ & $\begin{array}{l}\text { Three-arm mixed } \\
\text { methods pilot } \\
\text { study }\end{array}$ & $\begin{array}{l}\text { In hospital } \\
\text { (unspecified) }\end{array}$ & $30-37 w$ & 18 mothers & $\begin{array}{l}\text { Intervention arms } \\
\text { - mean } 23.1 \mathrm{y} \\
\text { and } 26 \mathrm{y} \text {, control } \\
\text { arm } 22.5 \mathrm{y} \\
11 \text { primips }\end{array}$ & Questionnaires & $\begin{array}{l}\text { Thematic } \\
\text { analysis }\end{array}$ & $\mathrm{B}$ \\
\hline $\begin{array}{l}\text { Tarus \& } \\
\text { Tjale } \\
\text { 2015[51] }\end{array}$ & $\begin{array}{l}\text { To understand } \\
\text { the mothers } \\
\text { lived } \\
\text { experience and } \\
\text { perception } \\
\text { towards 24- } \\
\text { hour Kangaroo } \\
\text { Mother Care } \\
\text { (KMC) during }\end{array}$ & $\begin{array}{l}\text { Johannesberg } \\
\text { Single site KMC } \\
\text { ward }\end{array}$ & Phenomenology & Unspecified & Unspecified & 9 mothers & $\begin{array}{l}18-38 y ; 3 \\
\text { primips }\end{array}$ & $\begin{array}{l}\text { Unstructured } \\
\text { interviews }\end{array}$ & $\begin{array}{l}\text { Colazzi's } \\
\text { method }\end{array}$ & $\mathrm{C}$ \\
\hline
\end{tabular}




\begin{tabular}{|c|c|c|c|c|c|c|c|c|c|c|}
\hline & $\begin{array}{l}\text { hospitalization } \\
\text { of their preterm } \\
\text { babies }\end{array}$ & & & & & & & & & \\
\hline $\begin{array}{l}\text { Flacking et } \\
\text { al } \\
2016[14]\end{array}$ & $\begin{array}{l}\text { Explore parents' } \\
\text { feelings of } \\
\text { emotional } \\
\text { closeness when } \\
\text { infant in NICU }\end{array}$ & $\begin{array}{l}\text { Sweden, England, } \\
\text { Finland } \\
\text { NICU level II/III } \\
\text { multi-centre }\end{array}$ & Qualitative & $\begin{array}{l}\text { In hospital } \\
\text { (unspecified) }\end{array}$ & Unspecified & 23 parents & Unspecified & $\begin{array}{l}\text { Emotional } \\
\text { closeness diary }\end{array}$ & $\begin{array}{l}\text { Thematic } \\
\text { network } \\
\text { analysis }\end{array}$ & $\mathrm{A} / \mathrm{B}$ \\
\hline $\begin{array}{l}\text { Martel et al } \\
\text { 2016[17] }\end{array}$ & $\begin{array}{l}\text { Describe the } \\
\text { establishment } \\
\text { of the father- } \\
\text { premature } \\
\text { infant } \\
\text { relationship } \\
\text { in a neonatal } \\
\text { unit }\end{array}$ & $\begin{array}{l}\text { Canada } \\
\text { neonatal nursery } \\
\text { single centre }\end{array}$ & Grounded theory & $\begin{array}{l}\text { In hospital } \\
\text { (unspecified) }\end{array}$ & $\begin{array}{l}32 w-36 w 6 d \\
\text { Mean 34w2d }\end{array}$ & 12 fathers & $\begin{array}{l}18-35+y ; 8 \text { first } \\
\text { time }\end{array}$ & $\begin{array}{l}\text { Semi- } \\
\text { structured } \\
\text { interviews, } \\
\text { daily journal }\end{array}$ & $\begin{array}{l}\text { Strauss and } \\
\text { Corbin's open, } \\
\text { axial, and } \\
\text { selective } \\
\text { coding. }\end{array}$ & $\mathrm{B} / \mathrm{C}$ \\
\hline $\begin{array}{l}\text { Nelson \& } \\
\text { Bedford } \\
\text { 2016[52] }\end{array}$ & $\begin{array}{l}\text { Describe } \\
\text { essential } \\
\text { elements of } \\
\text { mothering a } \\
\text { preterm infant } \\
\text { receiving }\end{array}$ & $\begin{array}{l}\text { USA } \\
\text { NICU level III } \\
\text { single centre }\end{array}$ & Phenomenology & $\begin{array}{l}\text { Around } \\
\text { discharge } \\
\text { (1 week } \\
\text { before or } \\
\text { after) }\end{array}$ & $24 w-29 w$ & 7 mothers & $\begin{array}{l}\text { 25-32y; } 4 \\
\text { primips }\end{array}$ & $\begin{array}{l}\text { Semi- } \\
\text { structured } \\
\text { interviews }\end{array}$ & $\begin{array}{l}\text { Colaizzi's } \\
\text { method }\end{array}$ & $\mathrm{A} / \mathrm{B}+$ \\
\hline
\end{tabular}




\begin{tabular}{|c|c|c|c|c|c|c|c|c|c|c|}
\hline & $\begin{array}{l}\text { NIDCAP care } \\
\text { in a NICU }\end{array}$ & & & & & & & & & \\
\hline $\begin{array}{l}\text { Phuma- } \\
\text { Ngaiyaye et } \\
\text { al 2016[53] }\end{array}$ & $\begin{array}{l}\text { Investigate } \\
\text { strategies for } \\
\text { supporting } \\
\text { maternal } \\
\text { newborn } \\
\text { bonding. }\end{array}$ & $\begin{array}{l}\text { Malawi } \\
\text { NICU unspecified } \\
\text { single centre }\end{array}$ & $\begin{array}{l}\text { Qualitative } \\
\text { exploratory }\end{array}$ & Unspecified & Unspecified & $\begin{array}{l}10 \text { mothers } \\
5 \text { midwives }\end{array}$ & Unspecified & $\begin{array}{l}\text { In-depth } \\
\text { interviews }\end{array}$ & $\begin{array}{l}\text { Content } \\
\text { analysis }\end{array}$ & $\mathrm{C}$ \\
\hline $\begin{array}{l}\text { Olsson et al } \\
\text { 2017[54] }\end{array}$ & $\begin{array}{l}\text { Describe } \\
\text { fathers' } \\
\text { experience } \\
\text { providing SSC } \\
\text { with their } \\
\text { premature } \\
\text { infant }\end{array}$ & $\begin{array}{l}\text { Sweden } \\
\text { NICU unspecified } \\
\text { multi-centre }\end{array}$ & $\begin{array}{l}\text { Qualitative } \\
\text { descriptive }\end{array}$ & $\begin{array}{l}\text { In hospital } \\
\text { (2-74d after } \\
\text { birth) }\end{array}$ & $25 w-35 w 3 d$ & 20 fathers & $\begin{array}{l}23-45 y, \text { median } \\
32 ; \\
14 / 20 \text { first time } \\
\text { fathers }\end{array}$ & $\begin{array}{l}\text { Semi- } \\
\text { structured } \\
\text { interviews }\end{array}$ & $\begin{array}{l}\text { Directed } \\
\text { qualitative } \\
\text { content } \\
\text { analysis }\end{array}$ & $\mathrm{B} / \mathrm{B}+$ \\
\hline $\begin{array}{l}\text { Maastrup et } \\
\text { al } \\
2017[55]\end{array}$ & $\begin{array}{l}\text { Explore parent's } \\
\text { readiness for } \\
\text { SSC, and the } \\
\text { overall meaning } \\
\text { of SSC for } \\
\text { parents in } \\
\text { extreme }\end{array}$ & $\begin{array}{l}\text { Denmark } \\
\text { NICU level IV } \\
\text { single centre }\end{array}$ & $\begin{array}{l}\text { Qualitative } \\
\text { descriptive }\end{array}$ & $\begin{array}{l}\text { In hospital } \\
\text { (25w6d- } \\
27 w 6 d)\end{array}$ & $23 w 6 d-27 w$ & $\begin{array}{l}11 \text { mothers } \\
5 \text { fathers }\end{array}$ & Unspecified & $\begin{array}{l}\text { Semi- } \\
\text { structured } \\
\text { interviews }\end{array}$ & $\begin{array}{l}\text { Thematic } \\
\text { analysis }\end{array}$ & $\mathrm{A} / \mathrm{B}-$ \\
\hline
\end{tabular}




\begin{tabular}{|c|c|c|c|c|c|c|c|c|c|c|}
\hline & $\begin{array}{l}\text { preterms below } \\
28 \mathrm{w} \mathrm{GA} .\end{array}$ & & & & & & & & & \\
\hline $\begin{array}{l}\text { Treherne et } \\
\text { al } \\
2017[15]\end{array}$ & \begin{tabular}{|l} 
Describe \\
parent's \\
perceptions of \\
closeness and \\
separation with \\
preterms in \\
NICU
\end{tabular} & $\begin{array}{l}\text { Canada } \\
\text { NICU level III } \\
\text { single centre }\end{array}$ & $\begin{array}{l}\text { Qualitative } \\
\text { descriptive }\end{array}$ & $\begin{array}{l}\text { In hospital } \\
(8-94 d)\end{array}$ & $24 w-33 w 3 d$ & $\begin{array}{l}13 \text { mothers } \\
7 \text { fathers }\end{array}$ & $\begin{array}{l}\text { Fathers mean age } \\
37.3 y, \\
\text { mothers mean } \\
\text { age } 32.2 y \text {; } \\
65 \% \text { first time } \\
\text { parent }\end{array}$ & $\begin{array}{l}\text { Voice recorded } \\
\text { via HAPPY } \\
\text { App }\end{array}$ & $\begin{array}{l}\text { Qualitative } \\
\text { content } \\
\text { analysis }\end{array}$ & $\mathrm{A} / \mathrm{B}+$ \\
\hline $\begin{array}{l}\text { Bujold et al } \\
2018[20]\end{array}$ & $\begin{array}{l}\text { Explore if and } \\
\text { how mothers } \\
\text { with infants in } \\
\text { the NICU, } \\
\text { perceive } \\
\text { expressing } \\
\text { human milk as } \\
\text { a closeness or } \\
\text { separation } \\
\text { experience. }\end{array}$ & $\begin{array}{l}\text { Canada } \\
\text { NICU level III } \\
\text { single centre }\end{array}$ & $\begin{array}{l}\text { Descriptive } \\
\text { qualitative cross- } \\
\text { sectional study }\end{array}$ & $\begin{array}{l}\text { In hospital } \\
\text { (avg 37d (5- } \\
\text { 94d) after } \\
\text { birth) }\end{array}$ & $23 w-32 w$ & 15 mothers & $\begin{array}{l}\text { 26-44y (mean } \\
32 \mathrm{y}) ; 10 \text { primips }\end{array}$ & $\begin{array}{l}\text { Voice recorded } \\
\text { via HAPPY } \\
\text { App }\end{array}$ & $\begin{array}{l}\text { Thematic } \\
\text { content } \\
\text { analysis }\end{array}$ & $\mathrm{A} / \mathrm{B}$ \\
\hline
\end{tabular}




\begin{tabular}{|c|c|c|c|c|c|c|c|c|c|c|}
\hline $\begin{array}{l}\text { Ettenberger } \\
\text { et al } \\
2018[56]\end{array}$ & $\begin{array}{l}\text { Measure the } \\
\text { impact of song } \\
\text { writing on } \\
\text { bonding, mental } \\
\text { wellbeing, } \\
\text { anxiety levels } \\
\text { and depressive } \\
\text { symptoms in } \\
\text { mothers of } \\
\text { preterm babies } \\
\text { in NICU. }\end{array}$ & $\begin{array}{l}\text { Colombia } \\
\text { NICU level III } \\
\text { single centre }\end{array}$ & Mixed-methods & $\begin{array}{l}\text { In hospital } \\
\text { (1-6 days } \\
\text { after birth) }\end{array}$ & $\begin{array}{l}28 \mathrm{w}-34 \mathrm{w} \\
(\text { mean } \\
31 \mathrm{w} 2 \mathrm{~d})\end{array}$ & 15 mothers & 14-36y; 8 primps & $\begin{array}{l}\text { Questionnaires } \\
\text { semi- } \\
\text { structured } \\
\text { interviews }\end{array}$ & $\begin{array}{l}\text { Thematic } \\
\text { analysis }\end{array}$ & B \\
\hline $\begin{array}{l}\text { Jabraeili et } \\
\text { al } \\
\text { 2018[57] }\end{array}$ & $\begin{array}{l}\text { Explore } \\
\text { maternal } \\
\text { emotional } \\
\text { caregiving } \\
\text { experiences in a } \\
\text { neonatal unit. }\end{array}$ & $\begin{array}{l}\text { Iran } \\
\text { NICU level II } \\
\text { single centre }\end{array}$ & Ethnography & $\begin{array}{l}\text { In hospital } \\
\text { (unspecified) }\end{array}$ & $\begin{array}{l}12 \text { term } \\
\text { infants and } 7 \\
\text { preterm } \\
\text { infants }\end{array}$ & 19 mothers & $\begin{array}{l}\text { 14-33y; parity } \\
\text { unspecified }\end{array}$ & $\begin{array}{l}\text { Observations, } \\
\text { interviews }\end{array}$ & $\begin{array}{l}\text { Roper and } \\
\text { Shapira's } \\
\text { (2000) coding } \\
\text { framework. }\end{array}$ & $\mathrm{A} / \mathrm{B}-$ \\
\hline $\begin{array}{l}\text { Logan \& } \\
\text { Dormire } \\
2018[58]\end{array}$ & $\begin{array}{l}\text { Study } \\
\text { experiences and } \\
\text { stressors of } \\
\text { fathers of pre- } \\
\text { terms less than } \\
28 \text { weeks }\end{array}$ & $\begin{array}{l}\text { USA } \\
\text { NICU level III } \\
\text { multi-centre }\end{array}$ & Phenomenology & $\begin{array}{l}\text { In hospital } \\
\text { (2weeks- } \\
\text { 3months) }\end{array}$ & $25 w-27 w$ & 7 fathers & $\begin{array}{l}\text { 25-46 yrs; } \\
\text { parity } \\
\text { unspecified }\end{array}$ & $\begin{array}{l}\text { Structured } \\
\text { interviews, } \\
\text { field notes }\end{array}$ & $\begin{array}{l}\text { Gadamerian } \\
\text { hermeneutic } \\
\text { method }\end{array}$ & $\mathrm{A} / \mathrm{B}$ \\
\hline
\end{tabular}




\begin{tabular}{|c|c|c|c|c|c|c|c|c|c|c|}
\hline & $\begin{array}{l}\text { during NICU } \\
\text { stay }\end{array}$ & & & & & & & & & \\
\hline $\begin{array}{l}\text { Makela et al } \\
\text { 2018[16] }\end{array}$ & $\begin{array}{l}\text { Identify and } \\
\text { understand how } \\
\text { parents develop } \\
\text { a close bond to } \\
\text { their infants in } \\
\text { the NICU }\end{array}$ & \begin{tabular}{|l} 
Finland \\
NICU level III \\
single centre
\end{tabular} & $\begin{array}{l}\text { Qualitative } \\
\text { descriptive }\end{array}$ & $\begin{array}{l}\text { In hospital } \\
\text { (median 8d } \\
\text { after birth) }\end{array}$ & $\begin{array}{l}26 w-41 w \\
\text { mean } 32 w 3 d\end{array}$ & $\begin{array}{l}18 \text { mothers } \\
5 \text { fathers }\end{array}$ & $\begin{array}{l}\text { Mothers age 27- } \\
38 \mathrm{y} \\
\text { Fathers age 27- } \\
42 \mathrm{y} ; \\
11 \text { primips }\end{array}$ & $\begin{array}{l}\text { Voice recorded } \\
\text { via HAPPY } \\
\text { App }\end{array}$ & $\begin{array}{l}\text { Inductive } \\
\text { thematic } \\
\text { analysis }\end{array}$ & $\mathrm{A} / \mathrm{B}$ \\
\hline $\begin{array}{l}\text { Medina et al } \\
2018[59]\end{array}$ & $\begin{array}{l}\text { Explore the } \\
\text { bonding process } \\
\text { of mothers } \\
\text { having } \\
\text { extremely } \\
\text { preterm infants } \\
\text { admitted in } \\
\text { NICU. }\end{array}$ & \begin{tabular}{|l} 
Spain \\
NICU level III \\
single centre
\end{tabular} & Phenomenology & $\begin{array}{l}\text { In hospital } \\
\text { (at least 30d } \\
\text { after birth) }\end{array}$ & $\begin{array}{l}24 w 5 d- \\
27 w 1 d\end{array}$ & 16 mothers & $\begin{array}{l}\text { 27-45y (mean } \\
\text { age } 34.4 y \pm S D \\
4,6 y) ; \\
\text { parity } \\
\text { unspecified. }\end{array}$ & $\begin{array}{l}\text { Focus groups } \\
\text { or interviews }\end{array}$ & $\begin{array}{l}\text { Gadamerian } \\
\text { hermeneutic } \\
\text { method }\end{array}$ & $\mathrm{A} / \mathrm{B}$ \\
\hline $\begin{array}{l}\text { Noren et al } \\
2018[60]\end{array}$ & $\begin{array}{l}\text { Describe } \\
\text { Swedish } \\
\text { mothers' } \\
\text { experiences of } \\
\text { KMC. }\end{array}$ & \begin{tabular}{|l|} 
Sweden \\
NICU level III \\
Multi-centre
\end{tabular} & $\begin{array}{l}\text { Qualitative } \\
\text { descriptive }\end{array}$ & $\begin{array}{l}\text { After } \\
\text { discharge } \\
(4 \mathrm{~m} \\
\text { corrected } \\
\text { age })\end{array}$ & $28 w-33 w 6 d$ & 13 mothers & $\begin{array}{l}25-42 \text { years; } \\
8 \text { primips }\end{array}$ & $\begin{array}{l}\text { Semi- } \\
\text { structured } \\
\text { interviews }\end{array}$ & $\begin{array}{l}\text { Qualitative } \\
\text { content } \\
\text { analysis }\end{array}$ & $\mathrm{B} / \mathrm{C}$ \\
\hline
\end{tabular}




\begin{tabular}{|c|c|c|c|c|c|c|c|c|c|c|}
\hline $\begin{array}{l}\text { Stefana et al } \\
2018[61]\end{array}$ & $\begin{array}{l}\text { Explore fathers' } \\
\text { emotional } \\
\text { experiences } \\
\text { with the birth } \\
\text { and NICU stay } \\
\text { of their preterm } \\
\text { babies and to } \\
\text { try to identify } \\
\text { father profiles. }\end{array}$ & $\begin{array}{l}\text { Italy } \\
\text { NICU level III } \\
\text { single centre }\end{array}$ & Mixed-methods & $\begin{array}{l}\text { In hospital } \\
\text { (unspecified) }\end{array}$ & $23 w^{4 d}-33 w^{5 d}$ & 20 fathers & $\begin{array}{l}\text { mean age 39,4y; } \\
70 \% \text { first time } \\
\text { father }\end{array}$ & $\begin{array}{l}\text { Semi- } \\
\text { structured } \\
\text { interviews, } \\
\text { observation, } \\
\text { questionnaire }\end{array}$ & $\begin{array}{l}\text { Investigator } \\
\text { triangulation } \\
\text { approach/ } \\
\text { thematic } \\
\text { content } \\
\text { analysis }\end{array}$ & $\mathrm{B} / \mathrm{B}+$ \\
\hline $\begin{array}{l}\text { Hearn et al } \\
2019[62]\end{array}$ & $\begin{array}{l}\text { Describe } \\
\text { fathers' } \\
\text { involvement, } \\
\text { confidence and } \\
\text { beliefs of their } \\
\text { preterm infants. }\end{array}$ & $\begin{array}{l}\text { USA } \\
\text { NICU unspecified } \\
\text { single centre }\end{array}$ & Grounded theory & \begin{tabular}{|l} 
After \\
discharge \\
$(4-5 y)$
\end{tabular} & Unspecified & 19 fathers & $\begin{array}{l}\text { Age 28-57yrs } \\
(\text { mean } 38.1 y)\end{array}$ & $\begin{array}{l}\text { Semi- } \\
\text { structured } \\
\text { interviews }\end{array}$ & $\begin{array}{l}\text { Thematic } \\
\text { analysis }\end{array}$ & B \\
\hline
\end{tabular}

Abbreviations: GA - gestational age; QA - quality appraisal; KMC - Kangaroo mother care; SSC - skin-to-skin care; VLBW - very low birth weight; NIDCAP - neonatal individualized developmental care and assessment program

\section{${ }^{1}$ Variations in grades reflect different reviewer opinions}


The initial coding frame comprised 15 codes, with a further 26 codes added by the reviewers during the data extraction process. Further synthesis led to three core interlinking themes and associated sub-themes being identified (see Table 4 for themes/subthemes data mapped to the individual studies).

Table 4: Themes/subthemes linked to individual studies

\begin{tabular}{|c|c|c|c|c|c|c|c|c|}
\hline Themes & \multicolumn{3}{|c|}{ Embodied Connections } & \multicolumn{2}{|c|}{ Inner Knowing } & \multicolumn{3}{|c|}{ Evolving Parental Role } \\
\hline Subthemes & $\begin{array}{l}\text { Inner recognition } \\
\text { and reciprocal } \\
\text { relating }\end{array}$ & $\begin{array}{l}\text { Bonding as a } \\
\text { family }\end{array}$ & $\begin{array}{l}\text { Retaining } \\
\text { presence in } \\
\text { absence }\end{array}$ & $\begin{array}{l}\text { Reassurance of } \\
\text { health }\end{array}$ & $\begin{array}{l}\text { Understanding } \\
\text { the process and } \\
\text { expectations }\end{array}$ & $\begin{array}{l}\text { Contributing to } \\
\text { wellness }\end{array}$ & $\begin{array}{l}\text { Caring and } \\
\text { advocating for } \\
\text { infant's needs }\end{array}$ & $\begin{array}{l}\text { Acknowledged } \\
\text { as a parent }\end{array}$ \\
\hline $\begin{array}{l}\text { Kavanaugh et } \\
\text { al 1997[38] }\end{array}$ & $\mathrm{X}$ & & & & & $\mathrm{X}$ & & \\
\hline Neu 1999[39] & $\mathrm{X}$ & & & & $\mathrm{X}$ & $\mathrm{X}$ & & $\mathrm{X}$ \\
\hline $\begin{array}{l}\text { Lupton \& } \\
\text { Fenwick } \\
\text { 2001[40] }\end{array}$ & $\mathrm{X}$ & & $X$ & & $\mathrm{X}$ & $\mathrm{X}$ & $\mathrm{X}$ & $X$ \\
\hline $\begin{array}{l}\text { Roller } \\
\text { 2005[41] }\end{array}$ & $\mathrm{X}$ & & & $\mathrm{X}$ & $\mathrm{X}$ & $\mathrm{X}$ & & \\
\hline $\begin{array}{l}\text { Johnson } \\
\text { 2007[23] }\end{array}$ & $\mathrm{X}$ & $\mathrm{X}$ & & & $\mathrm{X}$ & & $\mathrm{X}$ & $\mathrm{X}$ \\
\hline $\begin{array}{l}\text { Leonard \& } \\
\text { Mayers } \\
\text { 2008[22] }\end{array}$ & $\mathrm{X}$ & & & & $\mathrm{X}$ & $\mathrm{X}$ & & $X$ \\
\hline $\begin{array}{l}\text { Lundqvist et al } \\
\text { 2007[42] }\end{array}$ & $\mathrm{X}$ & $\mathrm{X}$ & & $\mathrm{X}$ & $\mathrm{X}$ & & $\mathrm{X}$ & $X$ \\
\hline $\begin{array}{l}\text { Orapiriyakul et } \\
\text { al 2007[43] }\end{array}$ & $\mathrm{X}$ & & & $\mathrm{X}$ & $\mathrm{X}$ & & & \\
\hline $\begin{array}{l}\text { Fegran et al } \\
2008[44]\end{array}$ & $\mathrm{X}$ & $\mathrm{X}$ & & $\mathrm{X}$ & $\mathrm{X}$ & & $\mathrm{X}$ & \\
\hline $\begin{array}{l}\text { Fenwick et al } \\
2008[19]\end{array}$ & $\mathrm{X}$ & & & $\mathrm{X}$ & $\mathrm{X}$ & & $\mathrm{X}$ & $X$ \\
\hline
\end{tabular}




\begin{tabular}{|c|c|c|c|c|c|c|c|c|}
\hline $\begin{array}{l}\text { Chang Lee et } \\
\text { al 2009[45] }\end{array}$ & & & $\mathrm{X}$ & $\mathrm{X}$ & $\mathrm{X}$ & $\mathrm{X}$ & & \\
\hline $\begin{array}{l}\text { Blomqvist et al } \\
2012[46]\end{array}$ & $\mathrm{X}$ & & & & & & $\mathrm{X}$ & \\
\hline $\begin{array}{l}\text { Arnold et al } \\
\text { 2013[47] }\end{array}$ & $\mathrm{X}$ & & & & $\mathrm{X}$ & & & \\
\hline $\begin{array}{l}\text { Guillaume et al } \\
2013[18]\end{array}$ & $\mathrm{X}$ & $\mathrm{X}$ & $\bar{X}$ & $\mathrm{X}$ & $\mathrm{X}$ & & & \\
\hline $\begin{array}{l}\text { Rossman et al } \\
2013[48]\end{array}$ & $\mathrm{X}$ & & & & & $\mathrm{X}$ & & \\
\hline $\begin{array}{l}\text { Flacking \& } \\
\text { Dykes } \\
2013[49]\end{array}$ & $\mathrm{X}$ & $\mathrm{X}$ & & & $\mathrm{X}$ & & & $X$ \\
\hline $\begin{array}{l}\text { Ettenberger et } \\
\text { al 2014[50] }\end{array}$ & $\mathrm{X}$ & & & & & $\mathrm{X}$ & $\mathrm{X}$ & \\
\hline $\begin{array}{l}\text { Tarus \& Tjale } \\
\text { 2015[51] }\end{array}$ & $\mathrm{X}$ & & & & & $\mathrm{X}$ & $X$ & $\mathrm{X}$ \\
\hline $\begin{array}{l}\text { Martel et al } \\
\text { 2016[17] }\end{array}$ & $\mathrm{X}$ & $\mathrm{X}$ & & $X$ & & & & $\mathrm{X}$ \\
\hline $\begin{array}{l}\text { Nelson \& } \\
\text { Bedford } \\
2016[52]\end{array}$ & & $\mathrm{X}$ & $X$ & & $\mathrm{X}$ & & $\mathrm{X}$ & $X$ \\
\hline $\begin{array}{l}\text { Phuma- } \\
\text { Ngaiyaye et al } \\
2016[53]\end{array}$ & $\mathrm{X}$ & & & $\mathrm{X}$ & & & & \\
\hline $\begin{array}{l}\text { Olsson et al } \\
\text { 2017[54] }\end{array}$ & $\mathrm{X}$ & $\mathrm{X}$ & & & $\mathrm{X}$ & $\mathrm{X}$ & & $\mathrm{X}$ \\
\hline $\begin{array}{l}\text { Flacking et al } \\
2017[14]\end{array}$ & $\mathrm{X}$ & $\mathrm{X}$ & & X & $\mathrm{X}$ & $\mathrm{X}$ & $\mathrm{X}$ & \\
\hline $\begin{array}{l}\text { Maastrup et al } \\
2017[55]\end{array}$ & $\mathrm{X}$ & & & & $\mathrm{X}$ & $\mathrm{X}$ & & $\mathrm{X}$ \\
\hline $\begin{array}{l}\text { Treherne et al } \\
2017[15]\end{array}$ & $X$ & $\mathrm{X}$ & & & $X$ & $X$ & $X$ & $X$ \\
\hline $\begin{array}{l}\text { Bujold et al } \\
\text { 2018[20] }\end{array}$ & & $X$ & & & & $\mathrm{X}$ & & \\
\hline $\begin{array}{l}\text { Ettenberger et } \\
\text { al 2018[56] }\end{array}$ & $\mathrm{X}$ & & & & & $\mathrm{X}$ & $\mathrm{X}$ & \\
\hline
\end{tabular}




\begin{tabular}{|c|c|c|c|c|c|c|c|}
\hline $\begin{array}{l}\text { Jabraeili et al } \\
\text { 2018[57] }\end{array}$ & $\mathrm{X}$ & & & $X$ & $\mathrm{X}$ & $\mathrm{X}$ & $\mathrm{X}$ \\
\hline $\begin{array}{l}\text { Logan \& } \\
\text { Dormire } \\
2018[58]\end{array}$ & $\mathrm{X}$ & $\mathrm{X}$ & & & & $X$ & \\
\hline $\begin{array}{l}\text { Makela et al } \\
\text { 2018[16] }\end{array}$ & $X$ & $\mathrm{X}$ & $X$ & & $\mathrm{X}$ & $\mathrm{X}$ & \\
\hline $\begin{array}{l}\text { Medina et al } \\
\text { 2018[59] }\end{array}$ & X & & & & $X$ & $X$ & \\
\hline $\begin{array}{l}\text { Noren et al } \\
2018[60]\end{array}$ & $\mathrm{X}$ & & & & $\mathrm{X}$ & X & $\mathrm{X}$ \\
\hline $\begin{array}{l}\text { Stefana et al } \\
\text { 2018[61] }\end{array}$ & $\mathrm{X}$ & & $\mathrm{X}$ & & & & \\
\hline $\begin{array}{l}\text { Hearn et al } \\
2019[62]\end{array}$ & $\mathrm{X}$ & & & & & & $\mathrm{X}$ \\
\hline
\end{tabular}

\section{Embodied connections}

The theme of 'embodied connections' concerns physical and embodied aspects of how parents formed emotional connections to their infants. Three sub-themes describe how parent-infant closeness was developed via a sense of embodied knowing and reciprocal actions ( inner recognition and reciprocal relating'), spending time as a family unit ('bonding as a family'), and how the use of strategies and artefacts enabled parents to feel connected while separated ('retaining presence in absence').

\section{Inner recognition and reciprocal relating}

Physical contact such as touching, holding, smelling, skin-to-skin/kangaroo care, or infant feeding was perceived as essential for parents to feel love and attachment towards their infants $[14-19,22,23,38,40,42-44,46-49,51,55-62]$; it provided a sense of 'realness' that the infant was theirs [14]. Mothers in Orapiriyakul et al's [43] study considered the effects to be cumulative, with increased contacts equated to 'greater' attachment. Whereas in Arnold et al's 
[47] study, half of the parents felt 'immediately bonded' when they first touched their infants. Studies that explored parents experiences of skin-to-skin/kangaroo care considered that this method of parent contact, rather than more distal means, e.g., holding, touching was the catalyst for a meaningful parent-infant connection [39-41, 54, 55]. A mother in Maastrup et al's study [55] reported how skin-to-skin contact enabled her to feel 'she has become my daughter' [M13], p.6. Similarly, fathers in Martel et al's study were reported to have experienced skinto-skin/kangaroo care as a 'positive emotional awakening' [17] p.6.

Infant's frailty and an intrinsic desire to protect their infant was reported to be a key motivator for increased physical contacts in five studies $[14,17,44,47,55]$. Some parents perceived touch to be an important means to transmit love to their infants $[17,40,53]$. However, there was also evidence of reciprocity, with infant responses to physical contacts serving to encourage further contact and to strengthen parent-infant emotional closeness. Other parents referred to how they felt emotionally close when their infant(s) recognised and responded to them through eye contact or gestures $[14-17,19,23,41,43,50,51,53,56,59]$; 'my baby gets to know me better because I am next to her' (p.203)[51]. This could include the infant turning towards their parent, as 'he recognises you by your voice or by smell' [59] p.328, or squeezing their parent's fingers $[16,17]$. One mother from Rollers [41] study exploring experiences of kangaroo care reported:

I think she's knowing me, she's feeling me, and you know, I think she can just tell the difference by my being so attached... I just think she knows I'm her mother. (p.214) 


\section{Bonding as a family}

An emotional connection with infants was stimulated via parent-to-parent/family interactions. For instance, mothers reported how positive affirmation and encouragement from their partners encouraged caretaking activities [18] and expressing breastmilk [20], and these activities were reported to facilitate a mother-infant connection and to help women develop a maternal role. One of the English mothers from Flacking et al's cross-cultural study reported:

I cleaned him and changed him more confidently $2^{\text {nd }}$ time. I did it while his father watched and I felt so, so, proud and for the first time maybe like a mum. My partner was very impressed with me!!!" (EM2, p.5)[14].

Similar views were expressed by fathers, where, for example, they described how their involvement in skin-to-skin, whilst perhaps due to encouragement and affirmation from their partners, was key to them forging an emotional connection with their infants [44]. Fathers in studies undertaken in Sweden, USA and Canada also emphasised how observing mothers interacting and bonding with their infants $[17,42,58]$ was an essential factor for them to develop their own emotional connection with their infant(s): 'I really wanted her to have a closer connection with my son than I did' [58] p. 158.

Parent-infant emotional connections were also formed through parents being able to spend uninterrupted private time together as a family [14-16, 23, 49, 52]. In some contexts, this could be facilitated by single room designs $[14,49]$. For other parents, this space was created through moving furniture and use of privacy screens to enable them to have 'time alone with my little boy' [23](p. 571), or when the neonatal unit was quiet [15]. Opportunities for family 
involvement with caregiving practices such as older children being in skin-to-skin contacte with their sibling, or being present when mothers' were expressing breastmilk were associated with positive impacts on familial relationships and wellbeing [20, 54].

\section{Retaining presence in absence}

There were various strategies used by parents to retain a sense of emotional connection with their infant when they were absent from the NICU. For instance, some parents would leave an item of perceived personal value with the infant such as a photo, toy, clothing, pacifier, or fabric with the parent's scent $[18,52,63]$. In Chang Lee et al's study [45] several mothers made audiotapes for nurses to play to their infants in their absence - one reported:

I made tapes when I missed him ...I wanted him to get to know my voice and me. I talked to him on the audiotape...It made me feel that I was with him. [M17, p.33]

Other examples related to mothers seeking information remotely, such as through regular (morning and evening) telephone calls to the NU [18], which helped parents to feel a greater sense of connection [40], and in one study [18] to facilitate breastmilk expression:

It's very good to have news by telephone....it takes 15 seconds but afterwards, you feel so much better....then pffff! I pump my milk and I fill the bottle' (m7, p.6)

Mothers in Taiwan have a lying-in period (one month) post-birth [45] and do not leave the house; this cultural practice meant they were unable to visit their infants in the NU in the early period post-birth. These mothers explained that partners and wider family members were key to providing news of their infant and were invaluable source of support to enable them to feel 
more emotionally connected with the baby [45].

\section{Inner knowing}

The theme of 'inner knowing' concerns cognitive, knowing facets of closeness. Two subthemes describe how emotional closeness was facilitated through knowledge about the infant's and mother's health ('reassurance of health') and an understanding of the processes and norms of neonatal care ('understanding the process and expectations').[10]

\section{Reassurance of health}

Parents' feelings of emotional closeness to their infant was inextricably linked to their infant's health status; positive signs of infant growth and development encouraged and enabled parents to feel emotionally connected to their infant. Reassurance of infant health could be achieved by receiving updates and information from staff, the reduction in the infant's need for medical intervention, and observations of infant's behaviours and responses $[14,16-19,41,43,45,53$, 61]. In Fenwick et al's [19] study, mothers receiving some 'certainty' about the infant's viability helped them to connect. Whereas for fathers in Lundqvist et al's [42] study, the relationship with their infant became stronger once the infant was moved out of the incubator. A strategy used by staff in Roller et al's study when mothers and infants were separated after birth was to bring the mother a photograph of her infant. One mother reported on the significance of seeing and hearing news of her daughter's health:

[They] brought pictures back [from the NU] ....I felt a lot better when they went down there. They said she's doing fine. They said she's breathing on her own and she's doing good. I was like, good, thank God. (p. 214)[41] 
In two of the studies undertaken with fathers, fathers reported how it was only once they were assured about their partner's health, did they start to feel emotionally connected to their infant $[18,44]$.

\section{Understanding the process and expectations}

Parent-infant emotional closeness was also reported to be intrinsically related to parents' understanding of what had happened to them, and what was happening to their infant. In one study, this concerned an awareness and understanding of the parent's own emotions and experiences - a Swedish mother from Flacking et al's cross-cultural diary study [14] reported:

This is a really difficult question. I don't know when it [feeling emotionally close] happened and when I started to feel it, but it's likely that it was about 1 week after birth because the first week was difficult with lots of tears and everything that had happened so quickly. So, I would say when everything has calmed down and you've had the time to understand what has happened and what you have been through" (SM3, p. 4-5)

In other studies, feelings of closeness were developed and augmented by parents' understanding of the physiological impacts of prematurity and the care/support their infant required [14, 40, 42, 43, 45]; 'being a mother is knowing your child' (p. 1015)[40]. Being in close physical proximity and observing their infants' responses enabled parents to pick up cues and to learn how to touch and interact with their infant $[14,15,18,19,22,23,39,41,44,45$, 49, 54, 55, 57]. A mother from a study undertaken in France [18] reported: 
It's important to understand her reactions, when she cries or seems nervous. If I don't manage to calm her, I feel like a bad mother who does not understand her child. It's important to understand and also to know what to do next (m15) p.6.

Acquiring an understanding of their infant's condition involved parents reading books on prematurity [45], observing, questioning and receiving feedback from staff $[15,18,19,40,52]$ and asking questions of other mothers [40]. It also involved an appreciation of the procedures, rules and regulations of the individual NU and an understanding of the purpose and nature of the technical devices used $[14,15,18,19,39,45,47,54]$.

\section{Evolving parental role}

The final theme of 'evolving parental role' relates to how parent's identities as a primary caregiver were manifested through different activities and actions. Three sub-themes describe how closeness was facilitated through parent's feeling that they were contributing to their infant's health ('contributing to wellness'), being able to provide direct care ('caring and advocating for infants needs') and being recognised and acknowledged as a parent ('acknowledged as a parent').

\section{Contributing to wellness}

Parent-infant connections were facilitated by parents' perceiving their behaviours and actions to have a direct impact on infant health and wellbeing. For instance, breastfeeding was highlighted as an important activity that provided mutual benefits $[14,16,38-40,45,48,59]$ : 
The most wonderful moment has probably been when the baby was on the breast for the first time. That feeling that the child gets nourishment from you makes us closer to each other. (FM7, p.4) [14].

Breastfeeding provided mothers with a unique role in their infant's lives, and their infants benefitted from the immunological and physiological benefits of breastfeeding. Similar sentiments were also expressed by mothers in Bujold et al's [20] study in relation to expressing breastmilk as it enabled them to feel like 'you're a mother' (p.4).

Parent-infant emotional closeness were also strengthened by parents observing how their actions such as breastfeeding, sending taped music to be played back, singing to their infant, physical touch, skin-to-skin/kangaroo care had visible and tangible benefits for their infants. These benefits could relate to infants gaining weight, requiring less medical intervention, and/or being visibly soothed or relaxed $[14,15,22,38,41,45,48,50,51,54-57,59,60]$; 'the nurses said that he was quiet when they played him the tapes' [M17, p. 333) [45]:

I'm happy that my presence is comforting to my baby. It makes me happy to know that I can help relieve some of her suffering (p.E8)[57]

In the study by Tarus \& Tjale [51], mothers referred to how close physical contact also enabled them to identify any changes or alterations to their infant's wellbeing, and to alert staff accordingly:

When providing KMC to the child, it is easy to see her if in case any changes like color or anything happens to her. It is easy to see. Even breathing you can hear the heartbeat 
when she is in your chest but in the incubator you won't be able to notice that earlier. In the incubator she is not all that close like in my chest' (Precious, p. 202)[51]

\section{Caring and advocating for infant's needs}

Providing basic parenting duties such as cleaning, bathing, feeding, washing, changing diapers, and dressing, was reported to have increased parent's feelings of connection and love [14-16, $19,23,40,42,44,46,51,52,56-58,60]:$

When we started to take care of the daughter more and more ourselves, the connection and love became stronger and stronger and still is!" (SM5, p.5)[14]

One father reported how being able to participate in feeding provided him with a special feeling of closeness' [46] p.1992, whereas for other fathers in the study by Lundqvist et al, caretaking was reported to have made them feel 'confirmed as a father' [42] p.13. A mother who took part in a singing intervention considered how singing to their infant made them feel they had a more active role in their infant's care [56]. Providing basic cares, or other means to interact with their infants such as clinical-related care (e.g., administering medications such as vitamins)[40], singing [50,56], or skin-to-skin/kangaroo mother care provided parents with a meaningful purpose to interact with their infants and through which they were able to 'get to know' and to 'discover who our little baby is' [15]; strengthening and consolidating positive parent-infant relationships:

Thanks to the sessions [music therapy] I learned how to get to know my baby better' (Participant MT7) no p.n. 'Because they helped me to get to know the expressions of my daughter. (Participant MT14)[50] 
A further way in which parents sensed feelings of emotional closeness with their infants was by advocating for their needs [19]. Women in studies undertaken in the USA and Spain [52, 59] referred to how knowing their infant's status enabled them to be more emotionally engaged with their 'infant's battles' [59] and in directing their infant's care. The knowledge that parents gathered through staff, clinical indicators and self-taught means enabled them to feel that they knew their infants 'best' [46]. In turn, they felt able to inform staff of their infant's needs during ward rounds $[14,15]$, and in negotiating 'the best care' [19] for their infants.

\section{Acknowledged as a parent}

A further way parents developed and experienced emotional closeness to their infants was through being acknowledged and valued as a parent by healthcare staff. This related to when parents felt that they had a level of control over providing care for their infants that was recognised and supported by staff, and which in turn was reported to have helped parents to connect to their infant $[15,17,19,22,40,42,49,51,52,54,57,60]$. One father in Olsson et al's study [54] stated that: 'it's sort of a good feeling when you feel able to do it by yourself' (father 14, p.e6). In addition, an Australian mother in Fenwick et al's [19] reflected on how she was able to provide care independently:

Like being able to come in and pick her up myself and sort of start managing it myself, making more decisions rather than waiting for the nurses. I think that's when I started feeling more like a mother (p.76). [19]

Mothers in Fenwick et al's study [19] referred to how they would specifically use technical jargon and remain 'polite' and 'remain pleasant' (p. 76) to present themselves as a capable and 
adept parent, who could be trusted to provide direct support to their infants. Whereas other parents referred to how engaging in skin-to-skin contact $[39,55,60]$ or direct cares $[19,62]$ helped them to learn about themselves as parents and provided a sense of parental competence which enabled them to, e.g., 'push on' and 'get our own way' with staff (p. 78)[19], to become more involved and confident in providing care, and/or adopting other areas of responsibility [55].

Other means of feeling acknowledged as a parent that helped facilitate parent-infant emotional connections was through having conversations with staff who knew their infant as well as they did [15], and via staff encouragement and affirmation of the mother-infant relationship:

[Nurse] made me realize that I know my little girl, and confidence in knowing makes each holding better than the last (p. 571)[23]

Fathers also provided some interesting reflections in terms of how being able to care for their infants on the unit, such as providing skin-to-skin contact, enabled them to have a more equal caregiving role [54]. The dedicated and uninterrupted time to provide care for their infant on the neonatal unit led them to feel more included and for a 'special bond' to be developed, particularly when compared to their experience of having a term infant $[54,62]$. A father from Hearn et al. study reported:

Unlike my first ones ... I wasn't really there in the hospital stay with them. But with the twins, I was there all the time. It's just a different kind of bond ... (James, p.85)[62] 


\section{Discussion}

This meta-ethnography adopted a salutogenic approach to identify what facilitates and enables parents' experiences of emotional closeness to their infants while cared for in a neonatal unit. Insights into emotional closeness were identified from 34 studies undertaken in 17 different countries. Reciprocal translation was used to identify and assess similar issues and concepts that concerned emotional closeness with this data synthesised into three overarching themes. First, 'embodied connections' concerns how emotional closeness was facilitated by physical contacts and reciprocal parent-infant actions, spending uninterrupted time as a family, and use of strategies and artefacts to enable parents to feel connected while separated from their infants. The second theme, 'inner knowing' describes the cognitive aspects of emotional closeness how closeness was enabled via knowledge about the infant's and mother's health, and knowledge and understanding of the processes and norms of neonatal care. The third theme, 'evolving parental role', concerns how emotional closeness was intertwined with parental identities of feeling they were contributing to infant health and development, able and competent in providing direct cares, and being recognised and acknowledged as a parent. Other factors such as poor infant and/or maternal health, poor parent-staff relationships, cultural factors and restricted resources are well known to influence parent-infants relationships [15]. We made a conscious choice to undertake a salutogenic and reciprocal based analysis to identify transferable strategies to optimise parent and infant wellbeing in a neonatal context.

Arguably, emotional closeness operates as an important precursor to parent-infant attachment, which is observable and measurable at the end of the first year [64]. Bowlby's evolutionary theory of attachment concerns how attachment is mutually innate, whereby infants use facial and vocal cues such as cries to promote interaction, and parents are biologically programmed to be receptive to these cues [65]. Bowlby's work has been highly influential in informing 
wider theories on parent-infant relationships. For instance, the Mutual Regulation Model relies on coregulation of parent and infant interactions that require the infant to possess a certain level of maturity and a repertoire of behavioural responses[66]. However, due to developmental and physiological immaturity and ill health, premature/sick infants' behavioural responses are not well developed, and they are less able to engage in mutually responsive interactions [67-69]. Parents are also often restricted in their level of involvement in caregiving and responsiveness to their infants due to medical interventions, neonatal routines, and emotional responses such as fear and distress [12]. Thus, the evolution of a parent-infant relationship and emotional closeness in a neonatal context is more challenging. Data from the included studies in this review suggests that the underlying mechanism promoting emotional closeness was reciprocity. Flacking et al [70] use the term reciprocity to describe a mutually satisfying parentinfant breastfeeding relationship, whereby parents of premature infants acknowledge their own needs and perceived needs of their infants. We extend this notion to suggest that emotional closeness is initiated and developed through reciprocal interactions between parents, infants, staff and wider family members, and influenced by spatial (use of space on the unit), physical (opportunities for physical contacts), cognitive (understanding their infant's prognosis, and rules of the NU), embodied (feeling, acting and being perceived as a parent) and health (of mothers and infants) related aspects. These insights thereby offer a specific perspective on the different pathways that can catalyze reciprocal parent-infant attunement and to facilitate emotional closeness.

Our synthesis found that parents felt emotionally connected when they engaged in various forms of physical contact with their infant (i.e., touch, skin-to-skin contact). In the past two decades growing evidence on the neurobiological underpinnings of parenting behaviour reveals the dynamic interplay that occurs between the neurological systems of parents and their 
newborns[71]. Infant behaviours precipitate physiological processes that contribute to the development of the infant-parent relationship, as well as stimulate parent's neurological system and promote parental well-being[72]. Visual and auditory cues emitted by infants activate regions of the parental brain implicated in parenting behavior[73, 74]. Moreover, infant behaviours and somatosensory stimuli, such as touch or skin-to-skin contact, can enhance sensitive parenting behaviours[75]. Skin-to-skin contact, one form of physical closeness, is associated with decreased parental cortisol and anxiety and better parental well-being[76]. Thus, it may be that physical contact with the infant in the NU might be particularly salient for emotional closeness.

Single room designs that enable parents and infant to be housed together 24-7 are associated with improved parent and infant outcomes [77]. However, our review also highlights how an innovative use of space on the unit (e.g., use of screens) that enables families to spend private and uninterrupted time together was important [15, 23]. Our study also highlights how some parents used artefacts, or technology such as audio recordings to have or maintain a connection with their infant when they could not be physically present. In the NU context parents may experience separation anxiety; that is unpleasant feelings and concerns about leaving their infant[78], and thus such strategies may enable parents to cope with separation. Parents have described the negative emotions they experience with departures from the NU, and NU nurses have observed these as well $[79,80]$. Moderate distress upon separation is considered normal and desirable, and an indicator of secure attachment, and studies have found that mothers of infants who have health problems may be more likely to report significant separation anxiety $[81,82]$. 
A further key finding was how parents need to feel valued and acknowledged as an important caregiver in their infant's life, whether this be through providing cares, and/or advocating for their infant's needs. As the reality for parents of premature/sick infants care is very different from their antenatal expectations, they require targeted and specialist education, support and opportunities to care for their infants[83]. Our findings reflect those of Thomson et al. [83] in that in order for parents to feel emotional close to their infants they need to be able to understand their/their infant's situation, have sufficient resources (personal, social, spatial etc) to be able to provide care for their infant, and to receive positive feedback and motivation from others (staff, families).

\section{Implications for practice}

Some of the key implications from this work are as follows. First this work reinforces some of the central tenets of family centred care in terms of the need for positive parent-staff partnership practices and parents being providing with early and ongoing education so they can become active decision-makers and advocates in the care of their infant [83]. Other implications concern how NU policies and models of care provision must foster and not limit parental presence. As privacy was a key factor in promoting emotional closeness, and not all settings have single family rooms, conditions to create shared spaces for dedicated family time should be prioritised.

Staff can encourage parents to develop strategies that help them maintain an emotional connection to their infant when they are separated. Communication technologies, such as webconferencing are now utilized in some units to allow parents to visualize and hear their infant from elsewhere. Audiotapes of parent's speaking, reading or singing are also being used to 
expose infants to appropriate forms of auditory stimulation and may have the added value of helping parents feel close to their infant when they are not present at the bedside.

Infant responsiveness to their parent was important for parent's emotional closeness. Recognition and acknowledgement that the development and evolution of the parent-infant relationship in a neonatal context is more challenging and specific support is vital. Neonatal nurses are well-positioned to facilitate parents learning to recognize and interpret subtle infant cues, so parents can recognize signs that their infant is responding to them during interactions. Staff should also be educated on the neurobiology of parenting, so they understand the importance of, value and promote physical proximity for the well-being of both infants and their parents.

\section{Strengths and limitations}

The strengths of this meta-syntheses are the comprehensive and inclusive search strategy. The team members were from a range of professional/academic backgrounds (e.g., psychology, nursing, midwifery, social sciences, neonatology), who had expertise in undertaking systematic reviews and/or qualitative syntheses thereby enhancing the rigour of this work. A broad approach was adopted whereby any study that provided a substantial contribution, for example a theme, concerned with emotional closeness was included. Less than half the included studies had an explicit focus on the parent-infant relationship; most described elements of emotional closeness as a by-product of other parent-related activities such as breastfeeding or skin-toskin/kangaroo care. However, as reflected in Table 4, as no single study contributed to all the sub-themes, these studies collectively enabled richer and more insightful insights into when, how and why emotional closeness could be facilitated. We also found similar insights from parents who experienced different care contexts and were of different parities; although only 
one of the studies had been undertaken a low-income context, which means the findings may not be generalisable to these settings. Futhermore, as ethnicity was often missing from the included studies, this was not reported. Further work could focus on what emotional closeness means within different care contexts, e.g., units with open bay, family rooms; settings, e.g., low or middle income countries; and with different groups of parents, e.g., different ethnic groups, vulnerable population groups, etc.

\section{Conclusion}

This systematic review using meta-ethnography methods adopted a salutogenic perspective to identify what facilitates and enables parents' experiences of emotional closeness to their infants while cared for in a neonatal unit. Three key themes are reported that highlight the multefacted nature of emotional closeness. 'Embodied connections' describes how emotional closeness was facilitated by reciprocal parent-infant interactions. 'Inner knowing' reflects how knowledge and understanding of their infant's health and NU procedures were powerful enablers to experiencing emotional closeness. Finally, the 'evolving parental role' concerns how it was vital for parents to feel they were contributing to infant health, to feel valued by others and to have their parental identity acknowledged. This work offers new insights into what constitutes parents-infant emotional closeness and offers practice based strategiews into how it can be facilitated during neonatal care. Further research to confirm or expand these issues within focused research studies and with different populations is needed.

\section{References}

[1] World Health Organisation. Preterm Birth 2018: https://www.who.int/news-room/factsheets/detail/preterm-birth (accessed 30 April 2020)

[2] Roque, A.T.F., Lasiuk, G.C., Radunz, V., Hegadoren, K. Scoping Review of the Mental Health of Parents of Infants in the NICU, J. Obstet Gynecol. Neonatal Nurs. 46(4) (2017) 57687.

[3] Al Maghaireh, D.F., Abdullah, K.L., Chan, C.M., Piaw, C.Y., Al Kawafha, M.M.. Systematic review of qualitative studies exploring parental experiences in the Neonatal Intensive Care Unit, J. Clin. Nurs. 25(19-20) (2016) 2745-56. 
[4] Moore, K.A.C., Coker, K., DuBuisson, A.B., Swett, B., Edwards, W.H. Implementing potentially better practices for improving family-centered care in neonatal intensive care units: successes and challenges, Pediatrics. 111(Supplement E1) (2003) e450-e60.

[5] Malusky, S. A concept analysis of family-centered care in the NICU, Neonatal Network. 24(6) (2005) 25-32.

[6] Raiskila, S., Axelin, A., Rapeli, S., Vasko, I., Lehtonen, L.. Trends in care practices reflecting parental involvement in neonatal care, Early Hum. Dev. 90(12) (2014) 863-7.

[7] Raiskila, S., Axelin, A., Toome, L., Caballero, S., Tandberg, B.S., Montirosso, R., et al. Parents' presence and parent-infant closeness in 11 neonatal intensive care units in six European countries vary between and within the countries, Acta Paediatr. 106(6) (2017) 87888.

[8] O'Callaghan, N., Dee, A., Philip, R.K. Evidence-based design for neonatal units: a systematic review, Matern. Health Neonatol. Perinatol. (2019) 5:6 doi:10.1186/s40748-0190101-0.

[9] Flacking, R., Breili, C., Eriksson, M. Facilities for presence and provision of support to parents and significant others in neonatal units, Acta Paediatr. 108(12) (2019) 2186-91.

[10] Tandberg, B.S., Flacking, R., Markestad, T., Grundt, H., Moen, A. Parent psychological wellbeing in a single-family room versus an open bay neonatal intensive care unit, PLoS One. 14(11) (2019) 0224488 doi: 10.1371/journal.pone.0224488

[11] Tandberg, B.S., Froslie, K.F., Flacking, R., Grundt, H., Lehtonen, L., Moen, A. ParentInfant Closeness, Parents' Participation, and Nursing Support in Single-Family Room and Open Bay NICUs, J. Perinat. Neonatal Nurs. 32(4) (2018) E22-E32.

[12] Flacking, R., Lehtonen, L., Thomson, G., Axelin, A., Ahlqvist, S., Moran, V.H., et al. Closeness and separation in neonatal intensive care, Acta Paediatr. 101(10) (2012) 1032-7.

[13] Campbell, S.B., Taylor, P. Bonding and attachment: theoretical issues, Semin.

Perinatol. 3(1) (1979) 3-13.

[14] Flacking, R., Thomson, G., Axelin, A. Pathways to emotional closeness in neonatal units - a cross-national qualitative study, BMC Pregnancy Childb. 16(1) (2016) 170.

doi: $10.1186 / \mathrm{s} 12884-016-0955-3$

[15] Treherne, S.C., Feeley, N., Charbonneau, L., Axelin, A. Parents' perspectives of closeness and separation with their preterm infants in the NICU. J. Obstet. Gynecol. Neonatal Nurs. 46(5) (2017) 737-47.

[16] Mäkelä, H., Axelin, A., Feeley, N., Niela-Vilén, H. Clinging to closeness: The parental view on developing a close bond with their infants in a NICU, Midwifery. 62 (2018) 183-8.

[17] Martel, M-J., Milette, I., Bell, L., Tribble, DS-C., Payot, A., Dowling, D. Establishment of the relationship between fathers and premature infants in neonatal units, Adv. Neonatal Care. 16(5) (2016) 390-8.

[18] Guillaume, S., Michelin, N., Amrani, E., Benier, B., Durrmeyer, X., Lescure, S., et al. Parents' expectations of staff in the early bonding process with their premature babies in the intensive care setting: a qualitative multicenter study with 60 parents, BMC Pediatrics. 13(1) (2013) 18. doi: 10.1186/1471-2431-13-18

[19] Fenwick, J., Barclay, L., Schmied, V. Craving closeness: a grounded theory analysis of women's experiences of mothering in the Special Care Nursery, Women Birth. 21(2) (2008) 71-85.

[20] Bujold, M., Feeley, N., Axelin, A., Cinquino, C., Dowling, D., Thibeau, S. Expressing human milk in the NICU, Adv. Neonatal Care. 18(1) (2018) 38-48.

[21] Rossman, B., Engstrom, J.L., Meier, P.P., Vonderheid, S.C., Norr, K.F., Hill, P.D. "They've walked in my shoes": mothers of very low birth weight infants and their experiences 
with breastfeeding peer counselors in the neonatal intensive care unit, J. Hum. Lact. 27(1) (2011) 14-24.

[22] Leonard, A., Mayers, P. Parents' lived experience of providing kangaroo care to their preterm infants, Health SA Gesondheid. 13(4) (2008) 16-28.

[23] Johnson, A.N. The maternal experience of kangaroo holding, J. Obstet. Neonatal Nurs. 36(6) (2007) 568-73.

[24] Antonovsky, A. The salutogenic model as a theory to guide health promotion, Health Promot. Int. 11(1) (1996) 11-8.

[25] France, E.F., Cunningham, M., Ring, N., Uny, I., Duncan, E.A., Jepson, R.G., et al. Improving reporting of meta-ethnography: the eMERGe reporting guidance, BMC Med. Res. Methodol. 19, 25 (2019). https://doi.org/10.1186/s12874-018-0600-0

[26] Noblit, G.W., Hare, R.D. Meta-ethnography: Synthesizing qualitative studies, Sage, 1988 .

[27] France, E.F., Ring, N., Thomas, R., Noyes, J. Maxwell M, Jepson R. A methodological systematic review of what's wrong with meta-ethnography reporting, BMC Med. Res. Methodol. 14 (2014) 119. doi: 10.1186/1471-2288-14-119

[28] Thomson, G., Flacking, R.F., Haslund, H., Rowe, J., George, K., Feeley, N., Schmied, V., De Coen, K., Provenzi, L., Roberts, M-J. Parents' experiences of emotional closeness to their infants in the neonatal unit: a meta-ethnography, PROSPERO (2018) CRD42018105088

[29] Gibbs, D., Boshoff, K., Stanley, M. Becoming the parent of a preterm infant: A metaethnographic synthesis, Br. J. Occup. Ther.. 78(8) (2015) 475-87.

[30] Gooding, J.S., Cooper, L.G., Blaine, A.I., Franck, L.S., Howse, J.L., Berns, S.D. Family support and family-centered care in the neonatal intensive care unit: Origins, advances, impact, Semin. Perinat., Elsevier, 2011.

[31] Walsh, D., Downe, S. Appraising the quality of qualitative research, Midwifery. 22(2) (2006) 108-19.

[32] Downe, S., Walsh, D., Simpson, L., Steen, M. Template for metasynthesis. Contact: sdowne@uclanacuk. 2009.

33] Thomson, G., Feeley, C., Moran, V.H., Downe, S., Oladapo, O.T. Women's experiences of pharmacological and non-pharmacological pain relief methods for labour and childbirth: a qualitative systematic review. Reprod. Health. 16(1) (2019) 71

https://doi.org/10.1186/s12978-019-0735-4

[34] Feeley, C., Thomson, G., Downe, S. Caring for women making unconventional birth choices: a meta-ethnography exploring the views, attitudes, and experiences of midwives. Midwifery 72 (2019) 50-9.

[35] Downe, S., Finlayson, K., Oladapo, O.T., Bonet, M., Gülmezoglu, A.M. What matters to women during childbirth: a systematic qualitative review. PLOS One. 13(4) (2018) e0194906.

[36] Campbell, R., Pound, P., Morgan, M., Daker-White, G., Britten, N., Pill, R. et al. Evaluating meta ethnography: systematic analysis and synthesis of qualitative research. Health Technol Assess. 15(43) (2011) 1-164.

[37] Toye, F., Seers, K., Allcock, N., Briggs, M., Carr, E., Barker, K. Meta-ethnography 25 years on: challenges and insights for synthesising a large number of qualitative studies. BMC Med Res Methodol. 14(1) (2014) 1-14.

[38] Kavanaugh, K., Meier, P., Zimmermann, B., Mead, L. The rewards outweigh the efforts: breastfeeding outcomes for mothers of preterm infants, J. Hum. Lact. 13(1) (1997) $15-21$.

[39] Neu, M. Parents' perception of skin-to-skin care with their preterm infants requiring assisted ventilation, J. Obstet. Gynecol. Neonatal Nurs. 28(2) (1999) 157-64. 
[40]. Lupton, D., Fenwick, J. 'They've forgotten that I'm the mum': constructing and practising motherhood in special care nurseries, Soc. Sci. Med. 53(8) (2001) 1011-21.

[41] Roller, C.G. Getting to know you: mothers' experiences of kangaroo care, J. Obstet. Gynecol. Neonatal Nurs. 34(2) (2005) 210-7.

[42] Lundqvist, P., Westas, L.H., Hallström, I. From distance toward proximity: fathers lived experience of caring for their preterm infants, J. Pediatr. Nurs. 22(6) (2007) 490-7. [43] Orapiriyakul, R., Jirapaet, V., Rodcumdee, B., Kiatlaekakul, S., Taboonpong, S., Suttharangsee, W. Struggling to get connected: the process of maternal attachment to the preterm infant in the neonatal intensive care unit, Thai Journal of Nursing Research. 11(4) (2007) 251.

[44] Fegran, L., Helseth, S., Fagermoen, M.S. A comparison of mothers' and fathers' experiences of the attachment process in a neonatal intensive care unit, J. Clin Nurs. 17(6) (2008) 810-6.

[45] Chang Lee, S-N., Long, A., Boore, J. Taiwanese women's experiences of becoming a mother to a very-low-birth-weight preterm infant: A grounded theory study. Int. J. Nurs. Stud. 46(3) (2009) 326-36.

[46] Blomqvist, Y.T., Rubertsson, C., Kylberg, E., Jöreskog, K., Nyqvist, K.H. Kangaroo Mother Care helps fathers of preterm infants gain confidence in the paternal role. J Adv. Nurs. 68(9) (2012) 1988-96.

[47] Arnold, L., Sawyer, A., Rabe, H., Abbott. J., Gyte, G., Duley, L. et al. Parents' first moments with their very preterm babies: a qualitative study, BMJ Open. 3(4) (2013) e002487 doi: 10.1136/bmjopen-2012-002487

[48] Rossman, B., Kratovil, A.L., Greene, M.M., Engstrom, J.L., Meier, P.P. "I Have Faith in My Milk" The Meaning of Milk for Mothers of Very Low Birth Weight Infants Hospitalized in the Neonatal Intensive Care Unit. J. Hum. Lact. 29(3) (2013) 359-65.

[49] Flacking, R., Dykes, F. 'Being in a womb'or 'playing musical chairs': the impact of place and space on infant feeding in NICUs. BMC Preg. Child. 13(1) (2013) 179 doi: $10.1186 / 1471-2393-13-179$

[50] Ettenberger, M., Odell-Miller, H., Cárdenas, C.R., Serrano, S.T., Parker, M., Llanos, S.M.C. Music therapy with premature infants and their caregivers in Colombia-A mixed methods pilot study including a randomized trial, Voices: A World Forum for Music Therapy. 14(2) (2014) 1-28.

[51] Tarus, T.K., Tjale, A.A. Mothers' experiences of Kangaroo Mother Care during hospitalisation of their preterm babies at an academic hospital in Johannesburg. Am. J. Nurs. Sci. 4 (2015) 200-6.

[52] Nelson, A.M., Bedford, P.J. Mothering a preterm infant receiving NIDCAP care in a level III newborn intensive care unit, Pediatr. Nurs. 31(4) (2016) e271-e82.

[53] Phuma-Ngaiyaye, E., Kalembo, F.W. Supporting mothers to bond with their newborn babies: Strategies used in a neonatal intensive care unit at a tertiary hospital in Malawi, Int. J. Nurs. Sci. 3(4) (2016) 362-6.

[54] Olsson, E., Eriksson, M., Anderzén-Carlsson, A. Skin-to-skin contact facilitates more equal parenthood-a qualitative study from fathers' perspective. Pediatr. Nurs. 34 (2017) e2e9.

[55] Maastrup, R., Weis, J., Engsig, A.B., Johannsen, K.L., Zoffmann, V. 'Now she has become my daughter': parents' early experiences of skin-to-skin contact with extremely preterm infants. Scand. J. Caring. Sci. 32(2) (2018) 545-53.

[56] Ettenberger, M., Ardila, Y.M.B. Music therapy song writing with mothers of preterm babies in the Neonatal Intensive Care Unit (NICU) - A mixed-method spilot study, Arts Psychother. 58 (2018) 42-52. 
[57] Jabraeili, M., Hassankhani, H., Negarandeh, R., Abbaszadeh, M., Cleveland, L.M. Mothers' Emotional Experiences Providing Care for Their Infants Within the Culture of an Iranian Neonatal Unit, Adv. Neonatal Care. 18(4) (2018) E3-E12.

[58] Logan, R.M., Dormire, S. Finding My Way A Phenomenology of Fathering in the NICU, Adv. Neonatal Care. 18(2) (2018) 154-62.

[59] Medina, I.M.F., Granero-Molina, J., Fernandez-Sola, C., Hernandez-Padilla, J.M., Avila, M.C., Rodriguez, M. Bonding in neonatal intensive care units: Experiences of extremely preterm infants' mothers, Women Birth. 31(4) (2018) 325-30.

[60] Norén, J., Nyqvist, K.H., Rubertsson, C., Blomqvist, Y.T. Becoming a mother Mothers' experience of Kangaroo Mother Care, Sex. Reprod. HealthC. 16 (2018) 181-5. [61] Stefana, A., Padovani, E.M., Biban, P., Lavelli, M. Fathers' experiences with their preterm babies admitted to neonatal intensive care unit: A multi-method study, J. Adv. Nurs. 74(5) (2018) 1090-8.

[62] Hearn, G., Clarkson, G., Day, M. The Role of the NICU in Father Involvement, Beliefs, and Confidence: A Follow-up Qualitative Study, Adv. Neonatal Care. 20(1) (2019) 80-89.

[63] Lee, J., Kang, J.C., Ji, E.S. Experiences of Mothers' Attachment in a Follow-Up Program Using Early Intervention for Low-Birth-Weight Infants, Asian Nursing Research. 13(3) (2019) 177-83.

[64] Benoit, D. Infant-parent attachment: Definition, types, antecedents, measurement and outcome. J. Paediatr. Child Health. 9(8) (2004) 541-5.

[65] J. Bowlby, Attachment and loss: Attachment. Basic Books, New York, 1969.

[66] Beeghly, M., Tronick, E. Early resilience in the context of parent-infant relationships: A social developmental perspective, Curr. Probl. Pediatr. Adolesc. Health Care. 41(7) (2011) 197-201.

[67] White-Traut, R., Norr, K.F., Fabiyi, C., Rankin, K.M., Li, Z., Liu, L. Mother-infant interaction improves with a developmental intervention for mother-preterm infant dyads, Infant Behav. Dev. 36(4) (2013) 694-706.

[68] Provenzi, L., Fumagalli, M., Bernasconi, F., Sirgiovanni, I., Morandi, F., Borgatti, R., et al. Very preterm and full-term infants' response to socio-emotional stress: The role of postnatal maternal bonding, Infancy. 22(5) (2017) 695-712.

[69] Korja, R., Latva, R., Lehtonen, L. The effects of preterm birth on mother-infant interaction and attachment during the infant's first two years, Acta Obstet. Gynecol. Scand. 91(2) (2012) 164-73.

[70] Flacking, R., Ewald, U., Nyqvist, K.H., Starrin, B. Trustful bonds: a key to "becoming a mother" and to reciprocal breastfeeding. Stories of mothers of very preterm infants at a neonatal unit, Soc. Sci. Med. 62(1) (2006) 70-80.

[71] Bornstein, M.H., Putnick, D.L., Rigo, P., Esposito, G., Swain, J.E., Suwalsky, J.T., et al. Neurobiology of culturally common maternal responses to infant cry, Proc. Natl. Acad. Sci. 114(45) (2017) E9465-E73.

[72] S. Porges, C.S. Carter. Mechanisms, mediators, and adaptive consequences of caregiving, in: S.L. Brown, R.M. Brown, L.A. Penner (Eds.), Moving beyond self-interest: Perspectives from evolutionary biology, neuroscience, and the social sciences, Oxford University Press, New York, 2012, pp. 53-74.

[73] Kim, P., Strathearn, L., Swain, J.E. The maternal brain and its plasticity in humans, Horm. Behav. 77 (2016) 113-23.

[74] Abraham, E., Hendler, T., Shapira-Lichter, I., Kanat-Maymon, Y., Zagoory-Sharon, O., Feldman R. Father's brain is sensitive to childcare experiences, Proc. Natl. Acad. Sci. 111(27) (2014) 9792-7. 
[75] L. Strathearn. Exploring the neurobiology of attachment, in: P. Fongay, L. Fonagy, L. Mayes, M. Target (Eds.), Developmental science and psychoanalysis, Routledge, London, 2018. pp. 117-40.

[76] Athanasopoulou, E., Fox, J.R. Effects of kangaroo mother care on maternal mood and interaction patterns between parents and their preterm, low birth weight infants: a systematic review, Infant Ment. Health J. 35(3) (2014) 245-62.

[77] Shahheidari, M., Homer, C. Impact of the design of neonatal intensive care units on neonates, staff, and families: a systematic literature review, J. Neonate. Nurs. 26(3) (2012) 260-6.

[78] Hock, E., McBride, S., Gnezda, M.T. Maternal separation anxiety: Mother-infant separation from the maternal perspective, Child Dev. (1989) 793-802.

[79] Erlandsson, K., Fagerberg, I. Mothers' lived experiences of co-care and part-care after birth, and their strong desire to be close to their baby, Midwifery. 21(2) (2005) 131-8.

[80] Feeley, N., Genest, C., Niela-Vilén, H., Charbonneau, L., Axelin, A. Parents and nurses balancing parent-infant closeness and separation: a qualitative study of NICU nurses' perceptions, BMC Pediatr. 16(1) (2016) 134. doi: 10.1186/s12887-016-0663-1

[81] Cooklin, A.R., Lucas, N., Strazdins, L., Westrupp, E., Giallo, R., Canterford, L., et al. Heightened maternal separation anxiety in the postpartum: The role of socioeconomic disadvantage, J. Fam. Issues. 35(11) (2014) 1497-519.

[82] Hsu, H-C. Antecedents and consequences of separation anxiety in first-time mothers: Infant, mother, and social-contextual characteristics, Infant Behav. Dev. 27(2) (2004) 113-33. [83] Thomson, G., Moran, V.H., Axelin, A., Dykes, F., Flacking, R. Integrating a sense of coherence into the neonatal environment. BMC Pediatr. 13(1) (2013) 84 doi: $10.1186 / 1471-$ 2431-13-84 